\title{
Cosmology with a Shock-Wave
}

\author{
Joel Smoller ${ }^{1, \star}$, Blake Temple $^{2, \star \star}$ \\ ${ }^{1}$ Department of Mathematics, University of Michigan, Ann Arbor, MI 48109, USA. \\ E-mail: smoller@umich.edu \\ 2 Institute of Theoretical Dynamics (Mathematical Physics) and Department of Mathematics, UC-Davis, \\ Davis, CA 95616, USA. E-mail: temple@math.ucdavi.edu
}

Received: 11 January 1999 / Accepted: 30 August 1999

\begin{abstract}
We construct the simplest solution of the Einstein equations that incorporates a shock-wave into a standard Friedmann-Robertson-Walker metric whose equation of state accounts for the Hubble constant and the microwave background radiation temperature. This produces a new solution of the Einstein equations from which we are able to show that the distance from the shock-wave to the center of the explosion at present time is comparable to the Hubble distance. We are motivated by the idea that the expansion of the universe as measured by the Hubble constant might be accounted for by an event more similar to a classical explosion than by the well-accepted scenario of the Big Bang.
\end{abstract}

\section{Introduction}

In the standard model for cosmology it is assumed that the Cosmological Principle holds: on the largest scale, the entire universe is expanding at a rate measured by the Hubble law, $[1,6,7,9,14,15]$. Hubble's Law correlates recessional velocities of galaxies with red-shifts. However, this correlation has only been verified for nearby galaxies, and it is an extrapolation to apply this law to the entire universe. Moreover, it follows from the Einstein equations, $[2,3,14]$, that if the universe is everywhere expanding, then every spacetime point can be traced back to a singularity in the past, a singularity from which the entire universe burst in an event referred to as the Big Bang. The Cosmolgical Principle is what forces the singularity into the standard Big Bang interpretation of the origin of the universe. In this paper we explore the possibility that Hubble's Law actually only measures a localized expansion of the universe, and not the expansion of the entire universe. We demonstrate the consistency of this possibility by constructing the simplest possible solution of the Einstein equations that accounts for the observed

\footnotetext{
* Supported in part by NSF Applied Mathematics Grant No. DMS-980-2370, and in part by the Institute of Theoretical Dynamics (ITD), UC-Davis.

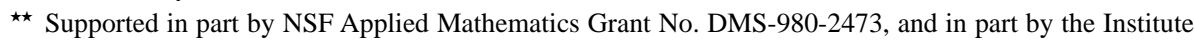
of Theoretical Dynamics (ITD).
} 
Hubble expansion rate and the correct microwave background radiation temperature, such that there is a shock-wave present at the leading edge of the expansion.

Our motivation is the idea that the expansion of the universe, as measured by the Hubble constant, might be the result of a large scale localized explosion that generated a shock-wave at the leading edge, not unlike a classical explosion into a static background, except on an enormously large scale. If this were true, then it would place our solar system in a special position relative to the center of the explosion, and this would violate the so-called Copernican Principle, at least on the scale at which the Hubble Law applies. The Copernican Principle is the statement that the earth is not in a "special place" in the universe. This principle justifies the standard cosmology based on the FriedmannRobertson-Walker (FRW) metric because the FRW metric is the unique metric that is consistent with the Einstein equations, and is homogeneous and isotropic about every point. The high degree of uniformity of the background microwave radiation in all directions, together with the directional independence of the redshifting of galaxies, provides the strongest support for the Copernican Principle. The idea that there is a shock-wave present at the leading edge of that portion of the universe where the Hubble constant applies, also violates another basic tenet of modern cosmology; namely, that we can meaningfully time reverse the continuum model all the way back to microseconds after the Big Bang. Indeed, it follows from the mathematical theory that shock-waves introduce a fundamental increase of entropy and consequent loss of information, [5, 13]. Thus, when a shock-wave is incorporated into cosmology, it becomes impossible to reconstruct the details of the early explosion from present data, at least at the level of the continuum model.

The simplest shock-wave model for cosmology is one in which the "expanding universe", inside the shock-wave, is modeled by the standard FRW metric of cosmology, and the spacetime on the outside is modeled by a Tolman-Oppenheimer-Volkoff (TOV) metric, (the general relativistic version of a static fluid sphere), such that the interface in between produces a spherically symmetric shock-wave solution of the Einstein equations propagating outward. (Such a shock-wave differs from a classical shock-wave because in addition to discontinuities in the fluid variables, there are discontinuities in the curvature of spacetime at the shock-wave.) The assumption that outside the shock-wave is a time-independent spherically symmetric solution is not unreasonable if one imagines that the spacetime before the explosion occurred took a long time getting into the preexplosion configuration; the assumption that an expanding FRW metric describes the spacetime behind the shock-wave is consistent with the fact that the galaxies appear to be uniformly expanding. In such a model one can interpret the shock-wave as thermalizing the radiation so as to maintain the uniform background radiation temperature behind the shock. (Of course, all of this implies that the model must be finely tuned to meet the physics.) In this paper we construct such a model assuming critical expansion, $(k=0)$, for the FRW metric, and what emerges is a new, essentially exact solution of the Einstein equations. We show that reasonable physical requirements on the TOV equation of state, (which can be interpreted as an entropy condition), put an interesting constraint on the possible position of the shock-wave relative to the center of the explosion. Using this, we derive precise estimates for the shock position at present time, as predicted by this model; that is, at the time in this model at which the Hubble constant and the value of the background radiation temperature agree with observed values. The constraint on the shock position can be interpreted as a length scale derived from the model, and this length scale is not determined by any adjustable parameters in the problem other than the experimentally determined values of the Hubble constant and the background radiation 
temperature. The constraint on the position of the interface arises because the interface is a true shock-wave. There is no similar constraint on the position of the interface in the well-known Oppenheimer-Snyder model, where the interface is a contact discontinuity, $[8,10]$.

In this paper we start with a critically expanding, $(k=0)$, FRW metric under the assumption that the equation of state agrees with the equation of state that applies in the standard model of cosmology after the time of the thermal uncoupling of matter from radiation. This uncoupling occurred at a temperature of approximately $4000^{\circ} \mathrm{K}$, at about 300, 000 years after the Big Bang in the standard model, [1,15]. In Sect. 3, we derive a system of ODE's that determine the TOV metrics that match the given FRW metric across a shock-wave interface, (Eqs. (5.3), (5.4) below). In fact, we derive the shock equations in the case of a general FRW metric, allowing for $k \neq 0$ and for general equations of state, (Eqs. (3.40), (3.41) below). In Sect. 4 we derive the FRW equation of state in terms of the cosmological scale factor, including a discussion of the FRW metric in the presence of both matter and radiation fields, assuming that the pressure due to matter is negligible, and that there is no thermal coupling between the fields. The results in Sect. 4 also apply for arbitrary $k$.

To obtain the ODE's for the TOV metric, we must rework the theory in $[10,12]$ where a given outer TOV metric is the starting point, instead of a given inner FRW metric which we require here. These ODE's, which are non-autonomous, simultaneously describe the TOV pressure $\bar{p}$ and the FRW shock position $r$, assuming conservation of energy and momentum and no delta function sources at the shock. (We let barred quantities refer to TOV variables and unbarred quantities to FRW variables, cf. [10].) We then derive a formula for the TOV energy density $\bar{\rho}$, (the only remaining undetermined variable in the TOV metric), which, together with solutions of the ODE's, determine the TOV solutions that match the given FRW metric across a shock-wave interface. The ODE's take a particularly simple form when the cosmological scale factor $R$ of the FRW metric is taken to be the independent variable instead of the usual TOV radial variable $\bar{r}$. In Sect. 5 we present a rather complete phase plane analysis of these equations and we prove that there exists a unique bounded orbit. This orbit describes the TOV pressure, but does not constrain either the initial shock position or the TOV energy density. We show that along this orbit, the pressure jump across the shock-wave has the property that the ratio of the TOV pressure to the FRW pressure at the shock is bounded between $1 / 9 \approx .1111$ and $\bar{\sigma}=\sqrt{17}-4 \approx .1231$, where the FRW pressure is supplied by the background radiation. Using this bound we obtain the following sharp upper and lower bounds for the distance that the shock-wave can propagate over and above the (geodesic) motion of the galaxies, as a function of "starting time", $R_{*}$. (Here $R=1$ denotes present time in the model, and we view the starting time $R_{*}<1$ as the earliest time at which the shock-wave solution has settled down to the point where our model applies; that is, as entropy increases, we expect shock-wave solutions to settle down to simple time asymptotic configurations, and we assume here that this time asymptotic solution agrees with our model from $R_{*}$ onward.) The inequality reads, (cf. Eq. (7.29) below),

$$
\frac{\left(2.62 \times 10^{-7}\right) T_{0}^{4}}{h_{0}^{2} H_{0}^{2}} \ln \left(\frac{1}{R_{*}}\right) \leq r^{2}-r_{*}^{2} \leq \frac{\left(2.65 \times 10^{-7}\right) T_{0}^{4}}{h_{0}^{2} H_{0}^{2}} \ln \left(\frac{1}{R_{*}}\right) .
$$

Here the distance $r$ is given in terms of the Hubble length

$$
H_{0}^{-1} \approx \frac{9.8}{h_{0}} \times 10^{9} \text { lightyears, }
$$


where

$$
H_{0}=100 h_{0} \mathrm{~km} \mathrm{~s}^{-1} \mathrm{mpc}^{-1} \text {, }
$$

and it is generally agreed that $h_{0}$ lies in the interval $.5 \leq h_{0} \leq .85$, [9]. For example, if we take $T_{0}=2.736 \approx 2.7^{\circ} \mathrm{K}, R_{*}=2.7 / 4000$, and $h_{0}=.55$, (a recently quoted value), the above estimate reduces to

$$
r^{2}-r_{*}^{2} \approx\left(\frac{.019}{H_{0}}\right)^{2}
$$

so that the distance our shock-wave has traveled between $R_{*}=2.7 / 4000$ and present time $R=1$, as predicted by this model, is approximately .019 times the Hubble length.

In the standard interpretation of the FRW metric in Cosmology, the galaxies are in freefall, and traverse geodesics $r=$ const. Thus we can interpret $r^{2}-r_{*}^{2}$ as the (squared) distance that the shock-wave travels over and above the motion due to freefall, a result of the fact that mass and momentum are driven across the shock-wave as it evolves outward. From this point of view it is a bit surprising that the quantity $r^{2}-r_{*}^{2}$ is independent of the starting position $r_{*}$.

Using the formula for the TOV energy density, we next prove that the minimal physical requirement $\rho>\bar{\rho}>\bar{p}>0$, (we take this as the entropy condition; in classical gas dynamics it is known that this is equivalent to the physical increase of entropy, [13]), places an additional constraint on the initial shock position $r_{*}$ that depends on the starting "time" $R_{*}$. We prove that once this constraint is met at one time, it is met at all succeeding times in the solution, and the density and pressure profiles are physically reasonable. We thus obtain the following bounds on the shock position at the present time as a function of background radiation temperature $T_{0}$, the Hubble constant $H_{0}$, and the starting value $R_{*} ;$ (cf. (7.35) and (7.36) below):

$$
\begin{aligned}
& r \geq H_{0}^{-1}\left\{\left(5.1 \times 10^{-4}\right) \frac{T_{0}^{2}}{h_{0}} \sqrt{\left.\ln \left(\frac{1}{R_{*}}\right)\right\}},\right. \\
& r \leq H_{0}^{-1} \sqrt{\frac{.76}{1+\left[\frac{\left(4.6 \times 10^{-7}\right) T_{0}^{4}}{h_{0}^{2} R_{*}^{2}}\right]} R_{*}+\left(2.6 \times 10^{-7}\right) \frac{T_{0}^{4}}{h_{0}^{2}} \ln \left(\frac{1}{R_{*}}\right)} .
\end{aligned}
$$

The maximum shock position is plotted in Fig. 2 for the case $T_{0}=2.7^{\circ} \mathrm{K}$, and $h_{0}=.55$. In Sect. 8 we compare these bounds to the analagous bounds one obtains in the case of pure radiation, thus making contact with the exact solution discussed in [11]. For example, at $T_{0}=2.7^{\circ} \mathrm{K}$ we obtain

$$
\frac{36 h_{0}}{H_{0}} \leq r \leq \frac{36 h_{0} \sqrt{1+2.5 R_{*}}}{H_{0}},
$$

cf. (8.7) below. (Of course, since we are neglecting the matter field, we do not have $\dot{R} / R=H_{0}$ at the same time when $T=T_{0}$ in the pure radiation model.) We note that in this case the distance from the shock position to the center of the explosion is significantly beyond the Hubble length.

In summary, starting with the idea that there might be a shock-wave that marks the outer boundary of the expansion that we measure by the Hubble constant, one's first reaction is that nothing quantitative could be said about the position of the shock without knowing details concerning the nature of the spacetime beyond the shock-wave, 
or details about the mechanism that might have created such an explosion in the first place. And to a large extent this must be true. But what we find interesting here is that this simplest shock-wave cosmological model, consistent with both the observed values of the Hubble constant and the background radiation temperature, contains within it unexpected constraints on the possible position of such a shock-wave, and the shock position is comparable to the Hubble length.

In conclusion, we ask whether our expanding universe could have evolved from the center of a great explosion that generated a shock-wave at its leading edge. If so it makes sense to wonder whether some of the far away objects that we observe in the nightime sky are possibly due to similar explosions that originated at other locations in spacetime. We now know that the scale of supernovae is not the largest scale on which classical explosions have occurred in the universe. Indeed, it was reported in a recent issue of Nature, that on May 7, 1998, a gamma ray explosion emanating from a faint galaxy known as GRB971214 erupted, and for two seconds the burst was more luminous than the rest of the universe combined. This is the largest explosion ever recorded, and redshifts place it at about 12 billion lightyears away. Moreover, conditions at the explosion were equivalent to those one millisecond after the Big Bang in the standard model. Thus we ask: could explosions such as this, or even greater than this, have given rise to our own "expanding universe"? Indeed, could we then observe other similar explosions in distant regions of spacetime beyond the expansion of our own universe, (that is, beyond the shock-wave that marks the edge of the expansion we measure by the Hubble constant)? We propose the shock-wave model presented in this paper as a natural and simple starting point for a further investigation of these issues. But independently of this, the model provides a new, essentially exact solution of the Einstein equations that we feel is interesting in its own right.

\section{Preliminaries}

According to Einstein's theory of general relativity, the gravitational field is described by a Lorenzian metric $g$ that satisfies the Einstein equations

$$
G=\frac{8 \pi \mathcal{G}}{c^{4}} T
$$

on 4-dimensional spacetime. Here $G$ is the Einstein curvature tensor, $\mathcal{G}$ denotes Newton's gravitational constant, $c$ denotes the speed of light, and $T$ is the stress energy tensor, the source of the gravitational field. In this paper we are concerned with FRW and TOV metrics, two spherically symmetric metrics which are exact solutions of (2.1) when $T$ takes the form of a stress tensor for a perfect fluid, namely

$$
T_{i j}=\left(p+\rho c^{2}\right) u_{i} u_{j}+p g_{i j},
$$

where $\rho$ denotes the mass-energy density, $p$ the pressure and $i, j=0, \ldots, 3$ denote indices of spacetime coordinates. The FRW metric is given by

$$
d s^{2}=-d(c t)^{2}+R^{2}(t)\left\{\frac{1}{1-k r^{2}} d r^{2}+r^{2}\left(d \theta^{2}+\sin ^{2}(\theta) d \phi^{2}\right)\right\},
$$

and the TOV metric is given by

$$
d \bar{s}^{2}=-B(\bar{r}) d(c \bar{t})^{2}+A(\bar{r})^{-1} d \bar{r}^{2}+\bar{r}^{2}\left(d \theta^{2}+\sin ^{2}(\theta) d \phi^{2}\right) .
$$


We write the TOV metric in barred coordinates so that it can be distinguished from the unbarred FRW coordinates when we do the matching of these two metrics below, cf. [10]. Substituting (2.3) into (2.1) yields the following FRW equations, [7,9, 14, 15]:

$$
\dot{R}^{2}=\frac{8 \pi \mathcal{G}}{3 c^{2}} \rho R^{2}-k,
$$

and

$$
p=-\rho-\frac{R \dot{\rho}}{3 \dot{R}}
$$

The unknowns $R, \rho$ and $p$ in the FRW equations are assumed to be functions of the FRW time $t$ alone, and "dot" denotes differentiation with respect to $t$. Assuming co-moving coordinates and substituting (2.4) into (2.1) yields the following TOV equations:

$$
\begin{gathered}
\frac{d M}{d \bar{r}}=4 \pi \bar{r}^{2} \bar{\rho} \\
-\bar{r}^{2} \frac{d \bar{p}}{d \bar{r}}=\frac{\mathcal{G}}{c^{2}} M \bar{\rho}\left(1+\frac{\bar{p}}{\bar{\rho}}\right)\left(1+\frac{4 \pi \bar{r}^{3} \bar{p}}{M}\right) A^{-1},
\end{gathered}
$$

and

$$
\frac{B^{\prime}}{B}=-2 \frac{\bar{p}^{\prime}}{\bar{p}+\bar{\rho}},
$$

where

$$
A=1-\frac{2 \mathcal{G} M}{c^{2} \bar{r}} .
$$

Here the unknown functions are the density $\bar{\rho}$, the pressure $\bar{p}$, and the total mass $M$, which are assumed to be functions of $\bar{r}$ alone, and prime denotes differentiation with respect to $\bar{r}$. In the next section we fix an FRW metric and derive equations for the TOV metrics that match the given FRW metric across a shock-wave interface at which the metric is only Lipschitz continuous, and across which conservation of mass and momentum hold, and at which there are no delta function sources. In [10] it is shown that the shock surface is given implicitly by

$$
M(\bar{r})=\frac{4 \pi}{3} \rho(t) \bar{r}^{3},
$$

and the metrics (2.3), (2.4) are identified via a coordinate transformation in which

$$
\bar{r}=R r .
$$




\section{Derivation of Equations}

In this section we derive equations that describe the time evolution of an outgoing spherical shock-wave interface together with an outer TOV metric, such that the shock surface matches a given FRW metric on the inside, and such that conservation of energy and momentum hold across the interface. The main point here is that we are assuming a given inner FRW metric, rather than assuming a given outer TOV metric as in [10,12]. Thus we seek a pair of equations that determine an outer TOV metric that matches a given FRW across a shock-wave interface. Rather than deriving the shock equations, we shall write them down and prove that solutions of these equations determine a shock-wave solution of the Einstein equations. (The reader can obtain a formal derivation of these equations by reversing the steps in the arguments below.)

Equation (3.10) in our first theorem below is the first equation in the pair of ODE's that we will work with.

Theorem 1. Assume that $\rho(t), p(t)$, and $R(t)$ solve the FRW system

$$
\begin{gathered}
\dot{R}=\sqrt{\frac{8 \pi \mathcal{G}}{3 c^{2}} R^{2} \rho-k,} \\
\dot{\rho}=-3 \frac{\dot{R}}{R}(\rho+p),
\end{gathered}
$$

over some interval

$$
I=\left(t_{1}, t_{2}\right)
$$

Assume that

$$
R(t)>0
$$

and that

$$
\dot{R} \neq 0
$$

on I. We assume WLOG, (by the choice of positive square root in (3.1)), that

$$
\dot{R}>0 \text {. }
$$

Assume further that $r(t)$ is a positive invertible function defined on $I$, and define $\bar{r}(t)$ on I by

$$
\bar{r}=R r .
$$

Define functions $M(\bar{r})$ and $\bar{\rho}(\bar{r}))$ by

$$
M(\bar{r}(t))=\frac{4 \pi}{3} \rho(t) \bar{r}(t)^{3},
$$

and

$$
\bar{\rho}(\bar{r})=\frac{M^{\prime}(\bar{r})}{4 \pi \bar{r}^{2}}
$$


where prime denotes differentiation with respect to $\bar{r}$. Assume, finally, that $r(t)$ satisfies

$$
\dot{r}=\frac{1}{R}\left(\frac{p-\bar{p}}{\rho+\bar{p}}\right) \frac{1-k r^{2}}{\dot{R} r},
$$

for some function $\bar{p}$, and that $\rho, p, M, \bar{\rho}$, and $\bar{p}$ are all positive valued functions on $I$. Then for all $t \in I$ we have,

$$
p=\frac{\gamma \theta \bar{\rho}-\rho}{1-\gamma \theta}
$$

where, [12],

$$
\begin{gathered}
\theta=\frac{A}{1-k r^{2}}, \\
A=1-\frac{2 \mathcal{G} M}{c^{2} \bar{r}},
\end{gathered}
$$

and

$$
\gamma=\frac{\rho+\bar{p}}{\bar{\rho}+\bar{p}} .
$$

That is, Theorem 1 implies that for a given FRW solution, (3.10) implies the conservation condition (3.11) when $M$ and $\bar{\rho}$ are defined by (3.8) and (3.9), (these latter two equations being the shock surface matching condition and the second TOV equation, respectively, $[10,12])$. Here dot denotes $\frac{d}{c t}$, and we assume $c=1$.

Proof. Differentiating (3.8) with respect to $c t$ and using (3.9) gives

$$
\dot{M}=\frac{d M}{d \bar{r}} \dot{\bar{r}}=4 \pi \bar{\rho} \bar{r}^{2} \dot{\bar{r}} .
$$

But (3.8) gives

$$
\dot{M}=\frac{4 \pi}{3} \dot{\rho} \bar{r}^{3}+4 \pi \rho \bar{r}^{2} \dot{\bar{r}}
$$

so from (3.15) and (3.16) we get

$$
\dot{\bar{r}}=\frac{R r}{3(\bar{\rho}-\rho)} \dot{\rho} .
$$

Using (3.2) in (3.17) gives

$$
\dot{\bar{r}}=-r \dot{R} \frac{p+\rho}{\bar{\rho}-\rho} .
$$

Using (3.7) and simplifying we have

$$
\dot{r} R+r \dot{R}=-\dot{R} r\left(\frac{\rho+p}{\bar{\rho}-\rho}\right) .
$$


Using (3.10) to eliminate $\dot{r}$ from (3.19) gives

$$
\frac{1-k r^{2}}{\dot{R}^{2} r^{2}}=-\left(\frac{p+\bar{\rho}}{\bar{\rho}-\rho}\right)\left(\frac{\rho+\bar{p}}{p-\bar{p}}\right) .
$$

We now use the identity

$$
\frac{1-k r^{2}}{\dot{R}^{2} r^{2}}=\frac{1}{1-\theta},
$$

which follows from (3.1) and (3.8). Indeed,

$$
\dot{R}^{2}=\frac{2 \mathcal{G}}{c^{2}} \frac{M}{\bar{r}^{3}} R^{2}-k .
$$

But (3.13) implies that

$$
\frac{2 \mathcal{G M}}{c^{2}}=(1-A) \bar{r},
$$

and using this gives

$$
\dot{R}^{2}=\frac{1-A}{r^{2}}-k
$$

or, (cf. [10,12]),

$$
r^{2} \dot{R}^{2}=-A+\left(1-k r^{2}\right) .
$$

Using (3.12) in (3.22) gives (3.21), as claimed.

Now using (3.21) in (3.20) yields

$$
\frac{1}{1-\theta}=-\left(\frac{p+\bar{\rho}}{\bar{\rho}-\rho}\right)\left(\frac{\rho+\bar{p}}{p-\bar{p}}\right) .
$$

Solving this for $p$ gives (3.11), where we have used (3.14). This completes the proof of Theorem 1.

For a given FRW metric, Theorem 1 tells us that the ODE (3.10) can be taken in place of the conservation constraint (3.11), and the reversal of the steps in the above proof can be regarded as a formal derivation of the ODE (3.10). We now record the following additional equations that follow from the hypotheses of Theorem 3.1.

Corollary 1. Assume that the hypotheses (3.1) through (3.10) of Theorem 3.1 hold. Then the following equations are valid:

$$
\begin{gathered}
\frac{\rho+p}{\rho-\bar{\rho}}=\frac{\gamma \theta}{\gamma \theta-1}, \\
\dot{\bar{r}}(\bar{\rho}+\bar{p})=\sqrt{1-k r^{2}}\left(\frac{\theta}{\sqrt{1-\theta}}\right)(p-\bar{p}), \\
\frac{\theta}{1-\theta}=\left(\frac{\rho+p}{\bar{\rho}-\rho}\right)\left(\frac{\bar{\rho}+\bar{p}}{p-\bar{p}}\right),
\end{gathered}
$$




$$
\begin{gathered}
\dot{\bar{r}}=\frac{\gamma \theta}{\gamma \theta-1} \sqrt{1-k r^{2}} \sqrt{1-\theta}, \\
r^{2} \dot{R}^{2}=-A+\left(1-k r^{2}\right), \\
\frac{1-k r^{2}}{r^{2} \dot{R}^{2}}=\frac{1}{1-\theta} .
\end{gathered}
$$

Proof. By Theorem 4.1, we know that (3.11) holds, and using this in the LHS of (3.23) gives the RHS of (3.23). Also, from (3.22),

$$
r \dot{R}=\sqrt{1-k r^{2}} \sqrt{1-\theta},
$$

and using this in (3.19) gives

$$
\dot{\bar{r}}=-\sqrt{1-k r^{2}} \sqrt{1-\theta}\left(\frac{\rho+p}{\bar{\rho}-\rho}\right) .
$$

Using (3.23) in (3.30) gives (3.26). From (3.11) we get

$$
p-\bar{p}=\frac{\gamma \theta \bar{\rho}-\rho}{1-\gamma \theta}-\frac{\bar{p}(1-\gamma \theta)}{1-\gamma \theta}=\frac{(\rho+\bar{p}) \theta)-(\rho+\bar{p})}{1-\gamma \theta},
$$

so

$$
\frac{p-\bar{p}}{\rho+\bar{p}}=\frac{\theta-1}{1-\gamma \theta} .
$$

To verify (3.24), we use (3.26) which we write in the form

$$
\dot{\bar{r}} \sqrt{1-k r^{2}} \frac{1}{\sqrt{1-\theta}} \frac{1-\theta}{\gamma \theta-1} \theta,
$$

and so from (3.31) we have

$$
\dot{\bar{r}}=\frac{\sqrt{1-k r^{2}}}{\sqrt{1-\theta}} \theta\left(\frac{p-\bar{p}}{\bar{\rho}-\bar{p}}\right) .
$$

Solving for $(\bar{\rho}+\bar{p}) \dot{\bar{r}}$ in (3.32) gives (3.24). Finally, to obtain (3.25), equate the RHS's of (3.30) and (3.32). Equations (3.27) and (3.28) have already been derived as (3.22) and (3.21) within the proof of Theorem 1.

Now assume that $\rho(t), p(t)$, and $R(t)$ solve the FRW system (3.1) and (3.2) for $t \in I$, and assume that the hypotheses (3.3) to (3.10) of Theorem 1 hold. We know from Theorem 1 that the conservation condition (3.11) also holds. We now find an equation for $\bar{p}(\bar{r})$, (Eq. (3.35) below), which guarantees that $\bar{p}$ solves the TOV equation (2.8), since then, in light of (3.9), the functions $\bar{\rho}(\bar{r}), \bar{p}(\bar{r})$, and $M(\bar{r})$ will then solve the TOV system as well. Defining $A(\bar{r})$ by (3.13), we can define the function $B(\bar{r})$ as a solution of the ODE, $[10,12]$,

$$
\frac{B^{\prime}(\bar{r})}{B(\bar{r})}=-\frac{2 \bar{p}^{\prime}(\bar{r})}{\bar{\rho}(\bar{r})+\bar{p}(\bar{r})}
$$


thus determining a TOV metric of the form (2.4). For this metric, co-moving coordinates are assumed, [15], and thus the 4-velocity $\mathbf{u}$ is given by

$$
u^{0}=\sqrt{B}, \quad u^{i}=0, i=1,2,3 .
$$

Note that we are free to choose any positive initial value for $B$ by suitable rescaling of the time coordinate $\bar{t}$. The next lemma demonstrates that if $\bar{p}$ satisfies Eq. (3.35) below, then as a consequence it also satisfies

$$
\dot{\bar{p}}=-\frac{\mathcal{G} M \dot{\bar{r}}}{c^{2} \bar{r}^{2}}(\bar{\rho}+\bar{p})\left(1+\frac{4 \pi \bar{p} \bar{r}^{3}}{M}\right) A^{-1},
$$

which is equivalent to the TOV equation (2.8).

Lemma 1. The hypotheses (3.1) to (3.10) of Theorem 3.1, together with the equation

$$
\dot{\bar{p}}=-\frac{\mathcal{G} M}{c^{2} \bar{r}^{2}}\left(\frac{1-k r^{2}}{\dot{R} r}\right)\left(\frac{A}{1-k r^{2}}\right)(p-\bar{p})\left(1+3 \frac{\bar{p}}{\rho}\right) A^{-1},
$$

imply that $\bar{p}(\bar{r})$ also solves the TOV equation (2.8).

Proof. By (3.28) we have

$$
\frac{\sqrt{1-k r^{2}}}{\dot{R} r}=\frac{1}{\sqrt{1-\theta}},
$$

and substituting this together with (3.12) into (3.35) gives

$$
\dot{\bar{p}}=-\frac{\mathcal{G} M}{c^{2} \bar{r}^{2}} \sqrt{1-k r^{2}}\left(\frac{\theta}{\sqrt{1-\theta}}\right)(p-\bar{p})\left(1+3 \frac{\bar{p}}{\rho}\right) A^{-1} .
$$

But using (3.24) and (3.8) in (3.36) we obtain

$$
\dot{\bar{p}}=-\frac{\mathcal{G} M}{c^{2} \bar{r}^{2}} \dot{\bar{r}}(\bar{\rho}+\bar{p})\left(1+\frac{4 \pi \bar{p} \bar{r}^{3}}{\frac{4 \pi}{3} \rho \bar{r}^{3}}\right) A^{-1},
$$

which, after dividing by $\dot{\bar{r}}$, directly implies the TOV equation (2.8).

We now introduce the system of ODE's whose solutions we analyze in subsequent sections:

Theorem 2. Assume that $\rho(t), p(t)$, and $R(t)$ satisfy the FRW equations (3.1) and (3.2) for $t \in I$, and that the other hypotheses (3.3) through (3.10) of Theorem 3.1 hold. Assume further that $(r(t), \bar{p}(t))$ solves the system of $O D E$ 's

$$
\begin{aligned}
& \dot{r}=\frac{1}{R}\left(\frac{p-\bar{p}}{\rho+\bar{p}}\right) \frac{1-k r^{2}}{\dot{R} r}, \\
& \dot{\bar{p}}=-\frac{\mathcal{G} M}{c^{2} \bar{r}^{2}} \frac{(p-\bar{p})\left(1+3 \frac{\bar{p}}{\rho}\right)}{\dot{R} r},
\end{aligned}
$$

for $t \in I$, where $\bar{r}, M(\bar{r})$, and $\bar{\rho}(\bar{r})$ are defined for $\bar{r}\left(t_{1}\right)<\bar{r}<\bar{r}\left(t_{2}\right)$ by (3.7), (3.8) and (3.9). Then $\bar{\rho}(\bar{r}), \bar{p}(\bar{r}), M(\bar{r})$ solve the TOV system (2.7), (2.8), and the conservation 
condition (3.11) holds for all $t \in I$. Furthermore, under these assumptions, the system (3.38), (3.39) is equivalent to the system

$$
\begin{aligned}
& \frac{d r}{d R}=\frac{1}{R\left(Q R^{2}-k\right)}\left(\frac{P-\bar{P}}{Q+\bar{P}}\right)\left(\frac{1-k r^{2}}{r}\right), \\
& \frac{d \bar{P}}{d R}=-\frac{1}{2} R \frac{(Q+3 \bar{P})(P-\bar{P})}{Q R^{2}-k},
\end{aligned}
$$

for $R\left(t_{1}\right)<R<R\left(t_{2}\right)$, where

$$
(Q, P, \bar{P})=\frac{8 \pi \mathcal{G}}{3 c^{2}}(\rho, p, \bar{p}),
$$

and all have the dimensions of inverse length squared.

Note that Eq. (3.39) is equivalent to (3.35) of Lemma 1, and the equivalence of system (3.38), (3.39) with (3.40), (3.41) follows because of the assumption $\dot{R} \neq 0$. This also implies that $P$ and $Q$ can be considered as functions of $R$, in which case Eqs. (3.40) and (3.41) close to form a well-defined nonlinear system of two ODE's. After solving (3.40), (3.41) the dependence of $R$ on $t$ can be recovered from (3.1). Thus for a given FRW metric and a given solution of (3.40), (3.41), the only variable remaining to be determined is the TOV energy density $\bar{Q}$. To obtain $\bar{Q}$, write (3.8) as

$$
2 \mathcal{G} M=Q \bar{r}^{3} \text {. }
$$

Writing (2.7) as

$$
\frac{d}{d \bar{r}}(2 \mathcal{G} M)=3 \bar{Q} \bar{r}^{2}
$$

differentiating (3.43), substituting into (3.44) and solving for $\bar{Q}$ gives

$$
\bar{Q}=\frac{1}{3 \bar{r}^{2}} \frac{d}{d \bar{r}}\left(Q \bar{r}^{3}\right)=Q+\frac{R r}{3} \frac{d}{d \bar{r}} Q .
$$

Note that if $Q$ decreases as the shock moves outward, (that is, the $\bar{r}$ position of the shock increases), then the second term in (3.45) is negative, and so $\bar{Q}<Q$, (the density behind the shock is greater than the density in front of the shock), as is the case for classical shock-waves in fluids, [13]. Note, however that the physically necessary condition $\bar{Q}>$ 0 , or the physically reasonable condition $\bar{Q}>\bar{P}$, is not guaranteed, and depends on the particular solution; cf. Sect. 6.

The final theorem of this section tells us that solutions of the ODE's (3.40), (3.41) do indeed determine exact shock-wave solutions of the Einstein equations.

Theorem 3. Assume that $\rho(t), p(t)$, and $R(t)$ satisfy the FRW equations (3.1) and (3.2) for $t \in I$, and that the hypotheses (3.3) through (3.9) of Theorem 3.1 hold. Assume further that $(r(R), \bar{P}(R))$ solve the system of ODE's (3.40), (3.41) for $R\left(t_{1}\right)<R<R\left(t_{2}\right)$. Assume that $Q, P, M, \bar{Q}, \bar{P}$, and A are all positive and that the shock speed in $F R W$ coordinates is less than the speed of light throughout the interval I. Then there exists a $C^{1,1}$ invertible coordinate transformation mapping $(t, r) \rightarrow(\bar{t}, \bar{r})$ of the form

$$
\begin{aligned}
& \bar{t}=\bar{t}(t, r), \\
& \bar{r}=\bar{r}(t, r) \equiv R(t) r,
\end{aligned}
$$


such that, under this identification, the resulting TOV metric matches the given FRW metric Lipschitz continuously across the shock surface $r=r(t)$. (The angular coordinates $\theta$ and $\phi$ are implicitly identified.) Moreover, the Lipschitz continuous metric defined by taking the FRW metric for $r<r(t)$ and the TOV metric for $r>r(t)$ defines a shock-wave solution of the Einstein equations ([4,7]), cf. [10,12]. In particular, the Rankine-Hugoniot jump conditions

$$
\left[T_{i j}\right] n^{i}=0, \quad j=0, \ldots, 3,
$$

hold across the shock surface; there are no "delta function sources" on the surface; there exists a regular $C^{1,1}$ coordinate transformation defined in a neighborhood of each point on the shock such that the metric components in the transformed coordinates, (which can be taken to be Gaussian normal coordinates), have smoothness level $C^{1,1}$; and the matched metric determines a weak solution of the Einstein equations in the sense of the theory of distributions, $c f$. [10].

Proof. The existence of the coordinate transformation is proved in [10] pp. 278-280 under the assumption that the shock surface is nowhere characteristic in the sense of (4.43) of that paper. Using (4.55) of the same reference, the non-characteristic condition can be re-written as

$$
\dot{\bar{r}} \neq-\frac{A}{\dot{R} r},
$$

which holds here because we assume that $A>0, r>0, \dot{R}>0$ and $\dot{\bar{r}}>0$. Since the normal vector $\mathbf{n}$ to the shock surface is non-null, (because we assume that the shock speed is less than the speed of light), and the functions $c(t, r)$ and $\bar{c}(\bar{t}, \bar{r})$ in Lemma 9 of [10] are here equal to $R(t) r$ and $\bar{r}$, respectively, it follows that the conclusions of Lemma 9, [10] are valid. Moreover, the conservation condition (3.11) is valid, and thus the argument in [10] that leads to (3.9) in this latter reference, implies that, under our hypotheses, condition (5.5) of Lemma 9, [10], follows from the conservation condition (3.11) above. (Note that the condition (2.20) of [10], assumed in that paper, is not needed here.) Since the conclusions of Theorem 3 are just a re-statement of the conclusions of Lemma 9, [10], the proof of Theorem 3 is now complete.

A remarkable aspect of the formulation of the shock equations given in (3.40) and (3.41) is that, if $Q$ and $P$ are given functions of $R$, (which can be obtained from the FRW equations once an equation of state is specified), then Eq. (3.41) for $\bar{P}$ uncouples from the $\bar{r}$ equation (3.40). Thus, in principle, one can solve system (3.40) and (3.41) by first solving the scalar non-autonomous equation (3.41) for $\bar{P}$.

Note that system (3.40), (3.41) includes, as a special case, the exact solutions first presented in [11]; that is, the case when $k=0$ and

$$
\begin{aligned}
& P=\sigma Q, \\
& \bar{P}=\frac{\bar{\sigma}}{3} \bar{Q},
\end{aligned}
$$

where

$$
\sigma=\frac{\bar{\sigma}(7+\bar{\sigma})}{3(1-\bar{\sigma})}
$$




\section{The Equation of State}

In this section we derive the FRW equation of state that we use to close the system (3.34) and (3.35). We consider the case when the equation of state for the FRW metric agrees with the equation of state in the standard model of cosmology after the time that the radiation in the universe uncoupled from thermal equilibrium with matter. This is an effort to account for the observed microwave backgound radiation level in our shockwave model. Our idea is that if the expanding universe arose from a great explosion, then one might conjecture that the expansion would have settled down to a uniform expansion by the time that this decoupling occurred. In the standard model of cosmology, the thermal uncoupling of radiation and matter occurred at about 300, 000 years after the Big Bang, at a temperature of about 4000 degrees Kelvin, $[1,15,9]$. Thus, we analyze our shock-wave model in the case that there is an energy density $\rho_{r}$ for the radiation, (which supplies a pressure $p_{r}=(1 / 3) \rho_{r}$ via the Stefan-Boltzmann law), and a separate energy density $\rho_{m}$ for the matter, which is assumed to exert a zero pressure $p_{m}=0$. Since the scale factor $R$ is the independent variable in our shock equations (3.34) and (3.35), we now obtain formulas for the FRW energy density and pressure as functions of $R$.

We start with the FRW equations in the form, [15],

$$
\dot{R}^{2}=\frac{8 \pi \mathcal{G}}{3 c^{2}} \rho R^{2}-k,
$$

and

$$
\frac{d}{d R}\left(\rho R^{3}\right)=-3 p R^{2} .
$$

(Again, we assume that "dot" denotes $d / d(c t)$.) We can rewrite (4.1) as

$$
\dot{R}^{2}=Q R^{2}-k,
$$

and Eq. (4.2) as

$$
\frac{d}{d R}\left(Q R^{3}\right)=-3 P R^{2},
$$

cf. (3.42). Now assume that the energy in the FRW system is in the form of pure radiation and matter alone, so that

$$
Q=Q_{r}+Q_{m},
$$

where $Q_{r}, Q_{m}$ denote the (appropriately scaled) energy density of radiation and matter, respectively. Further, assume that the pressure of radiation is given by the StefanBoltzmann Law, [15],

$$
P_{r}=(1 / 3) Q_{r},
$$

and that

$$
P_{m}=0,
$$

so that the uncoupling implies that

$$
P=P_{r}+P_{m}=P_{r}=(1 / 3) Q_{r} .
$$


Finally, assume that

$$
Q_{m}=\frac{\beta}{R^{3}},
$$

for some positive constant $\beta$, so that the total energy of matter within a (geodesically) expanding volume in the FRW metric remains constant. Substituting (4.6)-(4.9) into (4.4) gives

$$
\frac{\frac{d}{d R}\left(Q_{r} R^{3}\right)}{Q_{r} R^{3}}=-\frac{1}{R},
$$

which has the solution

$$
Q_{r}=\frac{3 \alpha}{R^{4}}
$$

for some positive constant $\alpha$. Then the FRW pressure is given by

$$
P=P_{r}=\frac{\alpha}{R^{4}}
$$

We conclude that the equation of state that applies to the FRW system under the assumption that radiation is uncoupled from matter is given by

$$
\begin{gathered}
Q=\frac{3 \alpha}{R^{4}}+\frac{\beta}{R^{3}}, \\
P=\frac{\alpha}{R^{4}} .
\end{gathered}
$$

Putting (4.13), (4.14) into Eqs. (3.40), (3.41) gives the system of ODE's

$$
\begin{aligned}
& \frac{d r}{d R}=\frac{R}{\left(3 \alpha+\beta R-k R^{2}\right)}\left(\frac{\alpha-\bar{P} R^{4}}{3 \alpha+\beta R+\bar{P} R^{4}}\right) \frac{\left(1-k r^{2}\right)}{r}, \\
& \frac{d \bar{P}}{d R}=-\frac{1}{2 R^{5}} \frac{\left(3 \alpha+\beta R+3 \bar{P} R^{4}\right)\left(\alpha-\bar{P} R^{4}\right)}{3 \alpha+\beta R-k R^{2}}
\end{aligned}
$$

Observe, again, that in this formulation, the second equation (4.16) uncouples from the first equation (4.15). Equations (4.13) and (4.14) together with the Stefan-Boltzmann law imply that the temperature of radiation is proportional to $1 / R$. Indeed, let $T \equiv T(R)$ denote the temperature of radiation. The Stefan-Bolzmann law relates the energy density of radiation $\rho_{r}$ to the temperature through the relation

$$
\rho_{r}=a T^{4}
$$

where

$$
a \approx 7.664 \times 10^{-15} \frac{\mathrm{erg}}{\mathrm{cm}^{3}}\left(K^{\circ}\right)^{4}
$$

Since $Q_{r}=\frac{8 \pi \mathcal{G}}{3 c^{2}} \rho_{r}$, we can write this as 


$$
Q_{r}=\hat{a} T^{4},
$$

where, [15],

$$
\hat{a}=\frac{8 \pi \mathcal{G} a}{3 c^{4}}
$$

defines the constant $\hat{a}$. Now by (4.11)

$$
\frac{3 \alpha}{R^{4}}=Q_{r}=\frac{8 \pi \mathcal{G}}{3 c^{4}} \rho_{r}=\frac{8 \pi \mathcal{G}}{3 c^{4}} a T^{4}=\hat{a} T^{4},
$$

we have the following lemma:

Lemma 2. The Stefan-Boltzmann law (4.6) implies that

$$
T=\left(\frac{3 \alpha}{\hat{a}}\right)^{1 / 4} \frac{1}{R} \text {. }
$$

\section{Restriction to $k=0$ - Phase Plane Analysis}

We now analyze system (4.15), (4.16) in the case of critical expansion when $k=0$. The case $k \neq 0$ will be considered in a subsequent paper. To start, note that in the case $k=0$, system (3.40), (3.41) reduces to

$$
\begin{aligned}
& \frac{d r}{d R}=\frac{1}{\left(Q R^{3}\right)}\left(\frac{P-\bar{P}}{Q+\bar{P}}\right)\left(\frac{1}{r}\right), \\
& \frac{d \bar{P}}{d R}=-\frac{1}{2} \frac{(Q+3 \bar{P})(P-\bar{P})}{Q R} .
\end{aligned}
$$

Assuming now that $Q$ and $P$ are given by (4.13), (4.14), and substituting these into (5.1), (5.2) yields the system

$$
\begin{aligned}
& \frac{d r}{d R}=\frac{R}{(3 \alpha+\beta R)}\left(\frac{\alpha-\bar{P} R^{4}}{3 \alpha+\beta R+\bar{P} R^{4}}\right) \frac{1}{r}, \\
& \frac{d \bar{P}}{d R}=-\frac{1}{2 R^{5}} \frac{\left(3 \alpha+\beta R+3 \bar{P} R^{4}\right)\left(\alpha-\bar{P} R^{4}\right)}{3 \alpha+\beta R},
\end{aligned}
$$

which is just system (4.15), (4.16) in the case $k=0$. Solutions of system (5.3) and (5.4) determine the shock position $r(R)$, (the position as measured by the radial coordinate of the FRW metric that is behind the shock-wave), together with the TOV pressure $\bar{P}(R)$ in front of the shock. The TOV pressure $\bar{p}(\bar{r})$ is then recovered from the solution $(r(R), \bar{P}(R))$ by inverting the equation $\bar{r}=\operatorname{Rr}(R)$ and using $\bar{P}=\frac{8 \pi \mathcal{G}}{3 c^{2}} \bar{p}$. The function $R(t)$ is obtained by solving the FRW equation (3.1) with $k=0$, and due to the scaling law for this equation, we are free to choose the scale factor $R_{0}$ such that $R_{0}=1$ at present time in the universe. The constants $\alpha$ and $\beta$ that determine $Q$ rescale with choice of $R_{0}$, and are determined from initial conditions for the FRW metric. For an FRW metric that models the expanding universe, we can take one of the two initial conditions as

$$
Q_{0}=3 \alpha+\beta=H_{0}^{2},
$$


where $H_{0}$ is the present value of the Hubble constant, cf. ([9]). Finally, the TOV energy density $Q$ is given by the formula in (3.45):

$$
\bar{Q}=\frac{1}{3 \bar{r}^{2}} \frac{d}{d \bar{r}}\left(Q \bar{r}^{3}\right)=Q+\frac{R r}{3} \frac{d}{d \bar{r}} Q .
$$

This simplifies under our special assumption (4.13) for $Q$. Indeed, by (4.13),

$$
\frac{d Q}{d \bar{r}}=\frac{d Q}{d R} \frac{d R}{d \bar{r}}=\frac{d}{d R}\left(\frac{3 \alpha}{R^{4}}+\frac{\beta}{R^{3}}\right)\left(r+R \frac{d r}{d R}\right)^{-1} .
$$

Since

$$
\frac{d \bar{r}}{d R}=\frac{d(R r)}{d R}=r+R \frac{d r}{d R}
$$

where $\frac{d r}{d R}$ is given by Eq. (5.1). Putting (5.1) and (5.7) into (5.6) and simplifying yields the following expression for the TOV energy density $\bar{Q}$ :

$$
\bar{Q}=Q-\frac{(4 \alpha+\beta R)\left(3+\frac{\beta}{\alpha} R+w\right)\left(3+\frac{\beta}{\alpha} R\right) \alpha r^{2}}{R^{4}\left\{\alpha r^{2}\left(3+\frac{\beta}{\alpha} R+w\right)\left(3+\frac{\beta}{\alpha} R\right)+(1-w) R^{2}\right\}},
$$

where

$$
w=\frac{\bar{P} R^{4}}{\alpha} .
$$

We conclude that each choice of constants $\alpha$ and $\beta$ and each choice of initial conditions for (5.3) and (5.4) determines a shock-wave solution of the Einstein equations, at each point where all the variables are positive. We now analyze solutions of system (5.3) and (5.4) in detail.

Substituting $w$ for $\bar{P}$ in (5.3), (5.4) yields the equivalent system

$$
\begin{aligned}
& \frac{d r}{d R}=\frac{R}{\left(3+\frac{\beta}{\alpha} R\right)}\left(\frac{1-w}{3+\frac{\beta}{\alpha} R+w}\right) \frac{1}{\alpha r} \\
& \frac{d w}{d R}=\frac{4 w}{R}\left(1-\frac{(1-w)\left(3+\frac{\beta}{\alpha} R+3 w\right)}{8\left(3+\frac{\beta}{\alpha} R\right) w}\right) .
\end{aligned}
$$

Now (5.12) is a non-autonomous scalar equation for $\bar{P}$ that uncouples from Eq. (5.11). In order to analyze Eq. (5.12), we rewrite (5.12) as an autonomous system, by setting

$$
S=\frac{1}{\alpha} R
$$

and

$$
u=\frac{1}{S}
$$


Substituting these into (5.12) and letting "dot" denote $d / d S$, we obtain the following autonomous system of two ODE's that is equivalent to Eq. (5.12):

$$
\begin{aligned}
\dot{u} & \equiv \frac{d u}{d S}=-u^{2} \\
\dot{w} & \equiv \frac{d w}{d S}=4 w u\left[1-\frac{(1-w)(\beta+3(1+w) u)}{8(\beta+3 u) w}\right] .
\end{aligned}
$$

We now analyze the phase plane associated with system (5.15), (5.16).

System (5.15), (5.16) has a line of rest points at $u=0$, and an isocline where $\dot{w}=0$. Setting the RHS of Eq. (5.16) equal to zero gives

$$
\left[1-\frac{(1-w)(\beta+3(1+w) u)}{8(\beta+3 u) w}\right]=0
$$

and solving this for $w$ gives

$$
w=\frac{(3 \beta+8 u)}{2 u}\left\{-1+\sqrt{1+\frac{4}{3} \frac{(\beta+3 u)}{(3 \beta+8 u)^{2}}} u\right\} \equiv \phi(u) .
$$

Thus the isocline is defined for $0<u<\infty$ by

$$
w=\phi(u) \text {. }
$$

Note first that when $\beta=0$, (the case of pure radiation), the isocline degenerates to

$$
\phi(u) \equiv \sqrt{17}-4=\bar{\sigma} .
$$

It is straightforward to verify that when $\beta=0$, the isocline is also a solution orbit of system (5.15), (5.16), and the special solution in [10] with $\sigma=1 / 3, \bar{\sigma}=\sqrt{17}-4$ corresponds to this orbit. We will see below that the special value $\bar{\sigma}=\sqrt{17}-4$ also is important in the case $\beta \neq 0$. The next theorem gives the qualitative behavior of the solution orbits of system (5.15), (5.16) in the (u,w)-plane when $\beta \neq 0$.

Theorem 4. Assume that $\beta \neq 0$. Then the following statements hold regarding solutions of system (5.15), (5.16)). (Here we define an orbit of system (5.15), (5.16) to be a function $w=w(u)$ such that $(u(S), w(1 / S)),(u=1 / S)$, is a solution of system (5.15), (5.16)):

(i) The isocline $w=\phi(u)$ defined by (5.18) is monotone increasing for $0<u<\infty$, and satisfies

$$
\begin{aligned}
& \lim _{u \rightarrow \infty} \phi(u)=\bar{\sigma} \equiv \sqrt{17}-4 \approx .1231 \ldots, \\
& \lim _{u \rightarrow 0} \phi(u)=\frac{1}{9} \approx .1111 \ldots, \\
& \lim _{u \rightarrow 0} \phi^{\prime}(u)=\frac{1-\left(\frac{1}{9}\right)^{2}-\frac{8}{9}}{9 \beta} \approx \frac{.01097 \ldots}{\beta}>0 .
\end{aligned}
$$

(ii) Orbits can only cross the isocline $w=\phi(u)$ once, from right to left in the $(u, w)$ plane, as $S$ increases, (see Fig. 1). 


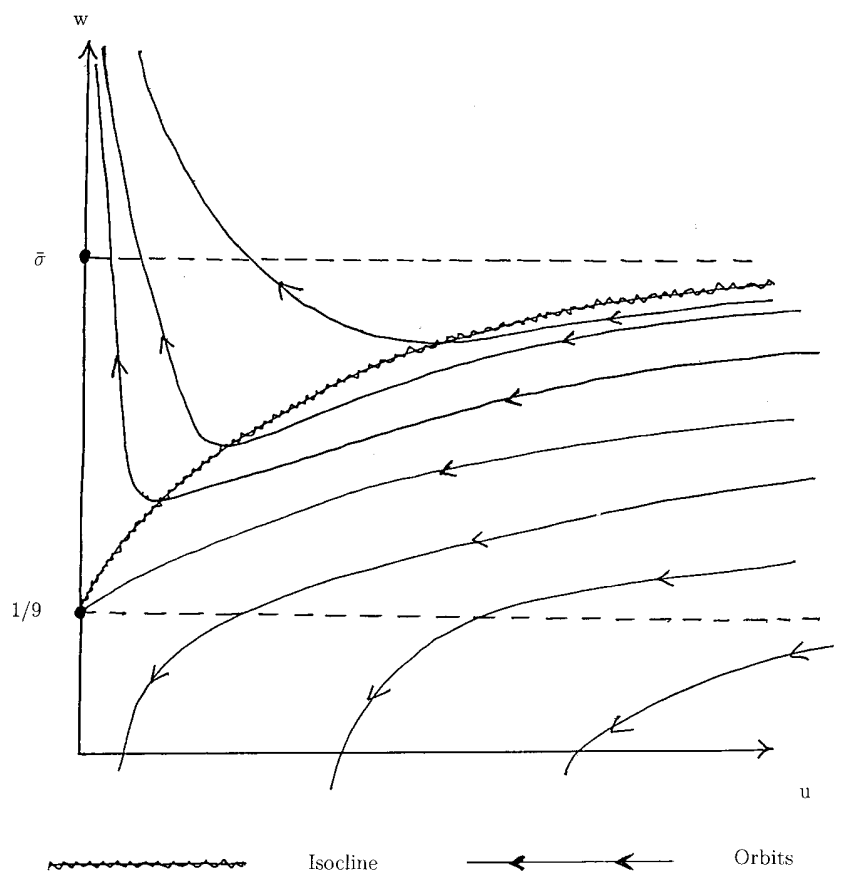

Fig. 1.

(iii) Along any orbit $w=w(u)$ we have

$$
\lim _{u \rightarrow \infty} w(u)=\bar{\sigma}
$$

(iv) There exists a unique orbit $w_{\text {crit }}(u)$ satisfying

$$
\lim _{u \rightarrow 0} w_{\text {crit }}(u)=\frac{1}{9}
$$

Moreover, all orbits $w=w(u)$ starting from initial conditions $\left(u_{0}, w_{0}\right)$ such that $w_{0}>\phi\left(u_{0}\right)$, (that is, starting above the isocline), satisfy

$$
\lim _{u \rightarrow 0} w(u)=\infty
$$

and all orbits starting from initial conditions $\left(u_{0}, w_{0}\right)$ such that $w_{0}<\phi\left(u_{0}\right)$, (starting below the isocline), satisfy

$$
\lim _{u \rightarrow 0} w(u)=-\infty
$$


Proof. To verify (5.21), we have

$$
\begin{aligned}
\lim _{u \rightarrow \infty} \phi(u) & =\lim _{u \rightarrow \infty} \frac{(3 \beta+8 u)}{2 u}\left\{-1+\sqrt{1+\frac{4}{3} \frac{(\beta+3 u)}{(3 \beta+8 u)^{2}}} u\right\} \\
& =\lim _{u \rightarrow \infty} \frac{(3 \beta+8 u)}{2 u}\left\{-1+\sqrt{1+\frac{1}{16}}\right\} \\
& =-1+\sqrt{17} \equiv \bar{\sigma} .
\end{aligned}
$$

We next show that $\phi(u)$ tends to $\bar{\sigma}$ monotonically from below as $u \rightarrow \infty$. Note that by (5.18), $w=\phi(u)$ is equivalent to

$$
0=\left[1-\frac{(1-w)(\beta+3(1+w) u)}{8(\beta+3 u) w}\right],
$$

which we rewrite as

$$
0=8(\beta+3 u) w-(1-w)[\beta+3(1+w) u] .
$$

Now differentiating (5.29) implicitly with respect to $u$ gives

$$
\begin{aligned}
8(\beta+3 u) \frac{d w}{d u}+24 w= & -[\beta+3(1+w) u] \frac{d w}{d u} \\
& +(1-w)\left[3+3 w+3 u \frac{d w}{d u}\right] .
\end{aligned}
$$

Simplifying (5.30) we obtain

$$
(9 \beta+24 u+6 u w) \frac{d w}{d u}=-3\left(w^{2}+8 w-1\right) .
$$

Now the roots of $w^{2}+8 w-1$ are

$$
\bar{\sigma} \equiv \sqrt{17}-4 \approx .1231, \quad \tilde{\sigma} \equiv-\sqrt{17}-4,
$$

and thus we conclude that, along the isocline $w=\phi(u)$,

$$
\begin{aligned}
& \frac{d w}{d u}>0 \text { if } w<\bar{\sigma} \\
& \frac{d w}{d u}<0 \text { if } w>\bar{\sigma}
\end{aligned}
$$

where we use the fact that $\phi(u)>0$ for all $u$. Thus, it suffices to show that $\phi(u) \neq \bar{\sigma}$ for any $u$ in order to conclude that $\phi^{\prime}(u) \neq 0$ for $0<u<\infty$. So assume for contradiction that $\phi(u)=\bar{\sigma}$. But solving for $u$ in (5.29) gives

$$
u=-\frac{\beta(9 w-1)}{(w-\bar{\sigma})(w+|\tilde{\sigma}|)},
$$

and thus $w=\bar{\sigma}$ leads to a contradiction unless $\beta=0$. We conclude that if $\beta \neq 0$, then $\phi(u)$ monotonically increases to $\bar{\sigma}$ as $u \rightarrow \infty$, thus proving (5.21). Statement (5.22) follows from (5.29), and (5.22) follows from (5.31). 
Statement (ii) follows because $\dot{w}=0$ only on $w=\phi(u), \dot{w}>0$ if $w>\phi(u)$, and since we have shown that $\phi^{\prime}(u)>0$, it follows that orbits can only cross the isocline from right to left in forward $S$-time.

To verify (iii), we show that all orbits tend in backward time, (increasing $u$ ), to $w=\bar{\sigma}$. To see this note that

$$
\begin{aligned}
\lim _{u \rightarrow \infty} \dot{w} & =\lim _{u \rightarrow \infty} 4 w u\left\{1-\frac{(1-w)\left(3+\frac{\beta}{u}+3 w\right)}{8\left(3+\frac{\beta}{u} w\right)}\right\} \\
& \approx 4 w u\left\{1-\frac{1-w^{2}}{8 w}\right\}
\end{aligned}
$$

where approximately means to leading order as $u \rightarrow \infty$. Now each orbit that starts above $w=\phi(u)$ decreases as $\mathrm{u}$ increases unless the orbit crosses the isocline, in which case the orbit increases from there on out as $u \rightarrow \infty$. It follows that orbits starting below $w=\phi(u)$ can never cross $w=\phi(u)$ at any value of $u$ larger than the initial value. Thus, since $\lim _{u \rightarrow \infty} \phi(u)=\bar{\sigma}$, all orbits must be bounded above in $w$ by the maximum of $\left\{\bar{\sigma}, w_{0}\right\}$, and bounded below by the minimum of $\left\{1 / 9, w_{0}\right\}$. But from (5.36), we must have that

$$
\lim _{u \rightarrow \infty}\left\{1-\frac{1-w^{2}}{8 w}\right\}=0 .
$$

Indeed, if not, then (5.36) implies that $|\dot{w}|$ tends to infinity as $u \rightarrow \infty$, which implies that $w$ is not bounded as $u \rightarrow \infty$, and this contradicts the above bounds. Since $\bar{\sigma}$ is the only positive root of $\left\{1-\frac{1-w^{2}}{8 w}\right\}$, we conclude from (5.37) that

$$
\lim _{u \rightarrow \infty} w=\bar{\sigma} .
$$

We now give the proof of (iv). From (5.15) and (5.16),

$$
\begin{aligned}
-\frac{d w}{d u} & =\frac{4 w}{u}\left[1-\frac{(1-w)(\beta+3(1+w) u)}{8(\beta+3 u) w}\right] \\
& \approx \frac{4 w}{u}\left[1-\frac{1-w}{8 w}\right],
\end{aligned}
$$

where approximate equality means to leading order as $u \rightarrow 0$. Now assume for contradiction that there exists an orbit $w=f(u)$ that is bounded in a neighborhood of $u=0$, but such that $\lim _{u \rightarrow 0} f(u) \neq 1 / 9$. The boundedness condition implies that (5.38) applies with errors that are bounded as $u \rightarrow 0$. That is,

$$
\begin{aligned}
-\frac{d w}{d u} & =\frac{4 w}{u}\left(1-\frac{1-w}{8 w}\right)+O(1) \\
& =\frac{9 w-1}{2 u}+O(1)
\end{aligned}
$$

where $O(1)$ denotes a constant that depends on the bounds for $w$ but is independent of $u$ as $u \rightarrow 0$. Integrating (5.40) leads to the estimate

$$
-\frac{9 w-1}{9 w_{0}-1}=\left(\frac{u_{0}}{u}\right)^{9 / 2}+O(1) e^{O(1)\left|u-u_{0}\right|},
$$


where $\left(u_{0}, w_{0}\right)$ are taken as initial data, $u_{0}>0$. But (5.41) implies that if $\lim _{u \rightarrow 0} w \neq$ $1 / 9$, then $w=f(u)$ is unbounded near $u=0$. From this we conclude that every orbit that is bounded as $u \rightarrow 0$ satisfies

$$
\lim _{u \rightarrow 0} w=1 / 9
$$

We now show that there exists at least one orbit such that $\lim _{u \rightarrow 0} w=1 / 9$. Note first that any orbit starting from initial data $\left(u_{0}, w_{0}\right)$ that lies on the isocline, $w_{0}=\phi\left(u_{0}\right)$, $u_{0}>0$, must lie above the isocline for all $0<u<u_{0}$ because we know that $\frac{d w}{d u}<0$ on this interval, and $\phi^{\prime}(u)>0$. Since the isocline decreases to $\bar{\sigma} \approx .1231>1 / 9$ as $u \rightarrow 0$, it follows that $w_{0}>1 / 9$ for initial data lying above the isocline, and hence $\lim _{u \rightarrow 0} w>1 / 9$ along an orbit starting from such initial data. But our argument above shows that when this happens, we must have $\lim _{u \rightarrow 0} w=+\infty$. We conclude that $\lim _{u \rightarrow 0} w=+\infty$ for any orbit starting from initial data above the isocline, $w_{0}>\phi\left(u_{0}\right)$. Similarly, if the initial data $\left(u_{0}, w_{0}\right)$ lies below the line $w=1 / 9$, that is, $w_{0}<1 / 9$, then also $w_{0}<\phi\left(u_{0}\right)$ because we have that $\phi(u)>1 / 9$. Thus from (5.38), $\frac{d w}{d u}>0$, and so it follows that $\lim _{u \rightarrow 0}<1 / 9$, and our argument above implies that $\lim _{u \rightarrow 0} w=-\infty$. We conclude that $\lim _{u \rightarrow 0} w=-\infty$ for any orbit starting from initial data below the line $w=1 / 9$; and $\lim _{u \rightarrow 0} w=+\infty$ for any orbit starting from initial data above the isocline, $w_{0}>\phi\left(u_{0}\right)$. Now consider all orbits emanating from initial data on some fixed vertical line $u=\epsilon>0$. Then if $w_{0}>\phi(\epsilon)$, we have $\lim _{u \rightarrow 0}=+\infty$; and if $w_{0}<1 / 9$, we have $\lim _{u \rightarrow 0}=-\infty$. So define

$$
w_{+}=\operatorname{Inf}\left\{w_{0}: \lim _{u \rightarrow 0} w=+\infty\right\},
$$

where the limit is taken along the orbit emanating from the point $\left(\epsilon, w_{0}\right)$. We now claim that the critical orbit emanating from initial condition $\left(\epsilon, w_{0}\right)$ satisfies $\lim _{u \rightarrow 0} w=1 / 9$. To see this note first that $w_{+} \geq 1 / 9$ because orbits below $w=1 / 9$ tend to $-\infty$ as $u \rightarrow 0$. We show next that the orbit emanating from $\left(\epsilon, w_{+}\right)$cannot tend to $w=+\infty$ as $u \rightarrow 0$. To see this, note that if $\lim _{u \rightarrow 0} w=+\infty$ along the crititcal orbit, then this must be true for all orbits starting in a neighborhood of $\left(\epsilon, w_{0}\right)$ as well. Indeed, if $\lim _{u \rightarrow 0} w=+\infty$, then at some positive value of $u$ we must have $w>\phi(u)$ along the critical orbit; and so by continuity, nearby orbits must also rise above the isocline at some $u>0$, and hence by above we know that $\lim _{u \rightarrow 0} w=+\infty$ along orbits sufficiently close to the critical orbit. But this contradicts the fact that $w_{-}$is a greatest lower bound. We conclude that we cannot have $\lim _{u \rightarrow 0} w=+\infty$ along the critical orbit. Similarly, we cannot have $\lim _{u \rightarrow 0} w=-\infty$ along the critical orbit because then nearby orbits would also satisfy $\lim _{u \rightarrow 0} w=-\infty$ since they would cross $w=1 / 9$ before $u=0$, and again this would contradict the fact that $w_{+}$is a greatest lower bound. Since we cannot have $\lim _{u \rightarrow 0} w=-\infty$ or $\lim _{u \rightarrow 0} w=+\infty$, it follows from (5.42) that the only alternative is that $\lim _{u \rightarrow 0} w=1 / 9$ along the critical orbit, as claimed.

We now show that the critical orbit is unique. To this end, rewrite Eq. (5.16) as

$$
\dot{w}=4 w u\left[1-\frac{(1-w)(\beta+3(1+w) u)}{8(\beta+3 u) w}\right] \equiv F(u, w) .
$$

Differentiating (5.44) with respect to $w$ gives

$$
\frac{d \dot{w}}{d w} \equiv \frac{\partial F}{\partial w}=4 u\left[1+\frac{6 w+\beta / u}{8(3+\beta / u)}\right]>0 .
$$


But (5.45) implies that the distance between orbits is increasing in forward time $S$, (that is, increasing as $u=1 / S$ decreases. Indeed,

$$
\left(w_{2}-w_{1}\right)=F\left(u, w_{2}\right)-F\left(u, w_{1}\right)=\frac{\partial F}{\partial w}\left(u, w_{*}\right)\left(w_{2}-w_{1}\right)>0
$$

if $w_{2}-w_{1}>0$. This implies that there cannot be two orbits that satisfy $\lim _{u \rightarrow 0} w=1 / 9$ since the distance between them would then tend to zero as $u \rightarrow 0$, contradicting (5.46). This finishes the proof of (iv), and thus the proof of the theorem is complete.

The salient properties of the phase plane for system (5.15), (5.16) are sketched in Fig. 1. Note that as $\beta \rightarrow 0$, the isocline moves up to the line $w=\bar{\sigma}$, (continuously, except for a jump from $1 / 9$ to $\bar{\sigma}$ at $u=0, \beta=0$ ). The isocline is a curve of absolute minima of orbits that cross the isocline, and the isocline, together with all orbits, tend to $w=\bar{\sigma}$ as $u \rightarrow \infty, R \rightarrow \infty$. Moreover, all orbits except the critical orbit tend to infinity as $u \rightarrow 0,(R \rightarrow \infty)$, and so the critical orbit is the only orbit bounded for all values of $R>0$. Along both the critical orbit and the isocline, the following apriori bounds hold for all $0<R<\infty$ :

$$
1 / 9 \approx .1111<w<\bar{\sigma} \approx .1231
$$

Note, however, that the critical orbit and the isocline do not coincide except in the limiting case $\beta=0$, in which case both reduce to the line $w=\bar{\sigma}$, which also can be identified with the special solution constructed in [10]. In particular, Fig. 1 describes how this special solution is imbedded in the larger class of solutions that allow for general initial data.

\section{Conditions for $\bar{Q}>0$ and $\bar{Q}>\bar{P}$}

In this section we obtain conditions which guarantee that $\bar{Q}>0$ and $\bar{Q}>\bar{P}$, physically reasonable conditions on the TOV energy density and pressure which are equivalent to the entropy inequality for shocks in classical gas dynamics. In particular, $\bar{Q}>\bar{P}$ guarantees that $\bar{Q}>0$ whenever the solution orbits of system (5.15), (5.16) satisfy $w \equiv \bar{P} R^{4} / \alpha>0$. We begin with the formula (5.9) for $\bar{Q}$, by asking that the RHS of (5.9) be positive. Using the formula

$$
Q=\frac{3 \alpha}{R^{4}}+\frac{\beta}{R^{3}}
$$

we see that $\bar{Q}>0$ is equivalent to

$$
\begin{array}{r}
\alpha\left\{\alpha r^{2}\left(3+\frac{\beta}{\alpha} R+w\right)\left(3+\frac{\beta}{\alpha} R\right)+(1-w) R^{2}\right\} \\
-(4 \alpha+\beta R)\left(3+\frac{\beta}{\alpha} R+w\right) \alpha r^{2}>0 .
\end{array}
$$

Solving (6.1) for $r^{2}$ leads to the following inequality that is equivalent to $\bar{Q}>0$ :

$$
\alpha r^{2}<\frac{(1-w) R^{2}}{3+\frac{\beta}{\alpha} R+w}
$$


Equation (6.2) implies that the condition $\bar{Q}>0$ puts a contraint on the maximum possible shock position at a given value of $R$. The following theorem implies that if the condition holds at some value $R=R_{*}$ in a solution of (5.15), (5.16), then it holds for all $R \geq R_{*}$ in that solution, so long as $0<w<1$ and $d w / d R<0$. Both of these conditions are satisfied along the critical orbit where $1 / 9<w<\bar{\sigma} \approx .1231$.

Lemma 3. Define the quantity \{\}$_{I}$ by

$$
\{\}_{I} \equiv\left\{\frac{(1-w) R^{2}}{3+\frac{\beta}{\alpha} R+w}-\alpha r^{2}\right\}_{I} .
$$

Then for any solution of (5.11), (5.12) we have

$$
\frac{d}{d R}\{\}_{I}>0
$$

at each point where

$$
0<w<\bar{\sigma}
$$

and

$$
\frac{d w}{d R}<0
$$

Lemma 3 implies that \{\}$_{I}$ is monotone increasing along any solution of (5.15), (5.16) that satisfies (6.6), and thus if (6.2) holds at a value $R=R_{*}$ in such a solution, then it must hold at all $R>R_{*}$; in particular it holds all along the critical orbit.

Proof. Starting with (6.3) we have

$$
\begin{aligned}
\frac{d}{d R} & \{\}_{I}=\frac{2(1-w) R}{3+\frac{\beta}{\alpha} R+w}-\alpha \frac{d r^{2}}{d R}+R^{2} \frac{d}{d R}\left\{\frac{1-w}{3+\frac{\beta}{\alpha} R+w}\right\}_{I I} \\
& =\frac{2(1-w) R}{3+\frac{\beta}{\alpha} R+w}-\frac{2(1-w) R}{\left(3+\frac{\beta}{\alpha} R+w\right)\left(3+\frac{\beta}{\alpha} R\right)}+R^{2} \frac{d}{d R}\{\}_{I I},
\end{aligned}
$$

where we have used (5.11). This simplifies to

$$
\frac{d}{d R}\{\}_{I}=\frac{2(1-w) R}{3+\frac{\beta}{\alpha} R+w}\left(\frac{2+\frac{\beta}{\alpha} R}{3+\frac{\beta}{\alpha} R}\right)+R^{2} \frac{d}{d R}\{\}_{I I} .
$$

Moreover,

$$
\begin{aligned}
\frac{d}{d R}\{\}_{I I} & =\frac{d}{d R}\left\{\frac{1-w}{3+\frac{\beta}{\alpha} R+w}\right\}_{I I} \\
& =\frac{\left(3+\frac{\beta}{\alpha} R+w\right)\left(-\frac{d w}{d R}\right)-(1-w)\left(\frac{\beta}{\alpha}+\frac{d w}{d R}\right)}{\left(3+\frac{\beta}{\alpha} R+w\right)^{2}} \\
& =\frac{\left(3+\frac{\beta}{\alpha} R+1\right)\left(-\frac{d w}{d R}\right)-(1-w) \frac{\beta}{\alpha}}{\left(3+\frac{\beta}{\alpha} R+w\right)^{2}} \\
& \geq-\frac{(1-w) \frac{\beta}{\alpha}}{\left(3+\frac{\beta}{\alpha} R+w\right)^{2}}
\end{aligned}
$$


where we have used (6.5) and (6.6). Using (6.9) in (6.8) and simplifying gives

$$
\frac{d}{d R}\{\}_{I} \geq \frac{(1-w)\left(4+\frac{\beta}{\alpha} R\right) R}{\left.\left(3+\frac{\beta}{\alpha} R+w\right)\right)\left(3+\frac{\beta}{\alpha} R\right)}>0 .
$$

This completes the proof of Lemma 3.

We now obtain a corresponding condition for $\bar{Q}>\bar{P}$. Using (5.10) and (6.5) we know

$$
\bar{P} \leq \frac{\alpha \bar{\sigma}}{R^{4}}
$$

Using this together with (5.9), we see that $\bar{Q}>\bar{P}$ will hold if

$$
\frac{R^{4} \bar{Q}}{\alpha}=\frac{3+\frac{\alpha}{\beta} R}{\alpha r^{2}\left(3+\frac{\beta}{\alpha} R\right)+\frac{R^{2}(1-w)}{3+\frac{\beta}{\alpha} R+w}}\{\}_{I} \geq \bar{\sigma} .
$$

Solving (6.12) for $\alpha r^{2}$ gives the equivalent condition

$$
\frac{1+\bar{\sigma}}{1-\frac{\bar{\sigma}}{3+\frac{\beta}{\alpha} R}} \alpha r^{2} \leq\{\}_{I I I}
$$

where

$$
\{\}_{I I I}=\frac{R^{2}(1-w)}{3+\frac{\beta}{\alpha} R+w} .
$$

Thus to get (6.13) it suffices to have

$$
\frac{1+\bar{\sigma}}{1-\frac{\bar{\sigma}}{3}} \alpha r^{2}=(1+\epsilon) \alpha r^{2} \leq\{\}_{I I I},
$$

where

$$
\epsilon=\frac{4 \bar{\sigma}}{3-\bar{\sigma}} .
$$

We conclude that $\bar{Q} \geq \bar{P}$ holds so long as

$$
\left\{\{\}_{I I I}-(1+\epsilon) \alpha r^{2}\right\} \geq 0 .
$$

Lemma 4. Define the quantity \{\}$_{I V}$ by

$$
\{\}_{I V} \equiv\left\{\{\}_{I I I}-(1+\epsilon) \alpha r^{2}\right\} .
$$

Then for any solution of (5.11), (5.12) we have

$$
\frac{d}{d R}\{\}_{I V}>0
$$

at each point where (6.5) and (6.6) hold. 
In particular, Lemma 4 implies that if (6.17) holds at a point $R_{*}$ in a solution of (5.11), (5.12) such that (6.5) and (6.6) hold for all $R \geq R_{*}$, then we conclude that (6.19) holds at all points $R \geq R_{*}$, and thus that $\bar{Q} \geq \bar{P}$ for all $R \geq R_{*}$. Thus (6.19) holds all along the critical orbit.

Proof. Differentiating we obtain

$$
\begin{aligned}
\frac{d}{d R}\{\}_{I V} & =\frac{2(1-w) R}{3+\frac{\beta}{\alpha} R+w}-(1+\epsilon) \alpha \frac{d r^{2}}{d R}+R^{2} \frac{d}{d R}\left\{\frac{1-w}{3+\frac{\beta}{\alpha} R+w}\right\}_{I I} \\
& =\frac{2(1-w) R}{3+\frac{\beta}{\alpha} R+w}-(1+\epsilon) \frac{2(1-w) R}{\left(3+\frac{\beta}{\alpha} R+w\right)\left(3+\frac{\beta}{\alpha} R\right)}+R^{2} \frac{d}{d R}\{\}_{I I} \\
& =\frac{2(1-w) R}{3+\frac{\beta}{\alpha} R+w}\left(\frac{2-\epsilon+\frac{\alpha}{\beta} R}{3+\frac{\alpha}{\beta} R}\right)+R^{2} \frac{d}{d R}\{\}_{I I} .
\end{aligned}
$$

Now using (6.9) in (6.20) and simplfying yields

$$
\frac{d}{d R}\{\}_{I V} \geq \frac{(1-w) R}{3+\frac{\beta}{\alpha} R+w} 3+\frac{\beta}{\alpha} R\left(4+\frac{\beta}{\alpha} R-2 \epsilon\right)>0,
$$

since $2 \epsilon<4$ and $w<1$. This concludes the proof of Lemma 4 .

We have proven the following theorem:

Theorem 5. Assume that (6.5) and (6.6) hold for all $R>R_{*}$ on a solution of (5.11), (5.12). Then \{\}$_{I}>0$ at $R=R_{*}$ is equivalent to $\bar{Q}>0$ at $R=R_{*}$, and implies that $\bar{Q}>0$ for all $R>R_{*}$; and if \{\}$_{I V}>0$ at $R=R_{*}$, then we must have $\bar{Q}>\bar{P}$ for all $R>R_{*}$. The condition \{\}$_{I}>0$ is equivalent to

$$
\alpha r^{2}<\frac{(1-w) R^{2}}{3+\frac{\beta}{\alpha} R+w},
$$

and the condition \{\}$_{I V}>0$ simplifies to

$$
\alpha r^{2}<\left(\frac{1-\bar{\sigma} / 3}{1+\bar{\sigma}}\right) \frac{(1-w) R^{2}}{3+\frac{\beta}{\alpha} R+w} .
$$

We end this section by showing that the constraint (6.22) implies that $A(\bar{r})>0$; that is, we show that the shock surface lies outside the Schwarzschild radius for the mass generated by the FRW mass behind the shock. Since

$$
A(\bar{r})=1-\frac{2 \mathcal{G} M(\bar{r})}{c^{2} \bar{r}^{2}},
$$

we require that

$$
2 \mathcal{G} M(\bar{r})<1,
$$

or, using the shock surface equation $M(\bar{r})=\frac{4 \pi}{3} \rho(t) \bar{r}^{3}$, this becomes $\bar{r}^{2}<Q^{-1}$, or $r^{2}<Q^{-1} R^{-2}$. Using (4.13), we need only show that

$$
\bar{r}^{2}<\frac{R^{2}}{3 \alpha+\beta R} .
$$

However, this is clearly implied by (6.22). 


\section{Estimates for the Shock Position}

In this section we take system (5.11), (5.12) as a simple cosmological model in which the FRW metric behind the shock-wave at position $r$ is assumed to model the expanding universe. Given this, we now estimate the position of the shock-wave in the present universe as determined by this model. In this model, the expanding universe is modeled by an FRW, $(k=0$,$) metric in which the energy density Q$ and pressure $P$ are given by (4.13), (4.14), that is, the same as that assumed in the standard cosmological model after the time of thermal decoupling of matter with radiation, (approximately 300,000 years after the Big Bang in the standard model, [15]). The FRW metric is assumed to have been created behind a radially expanding shock-wave due to a great explosion into a static, spherically symmetric universe modeled by a TOV metric. Given these assumptions, we have shown that conservation of energy at the shock then implies that the position $r$ of the shock-wave is determined by Eq. (5.11), where $r$ is the radial coordinate in the FRW universe behind the shock. Equation (5.11) is coupled to Eq. (5.12) for the TOV pressure $\bar{P}$, and the TOV energy density $\bar{Q}$ is then given by the formula (5.9). In this section we assume that $w=R^{4} \bar{P} / \alpha$ lies on the critical orbit $w=w_{\text {crit }}(S),\left(S=\frac{R}{\alpha}\right)$. (This is justified by the fact that, according to Theorem 4 , this is the only orbit bounded for all $R$, and all orbits are asymptotic to this one as $R \rightarrow 0$.) By Theorem $4, w$ ranges between $\bar{\sigma}$ and $1 / 9$ along the critical orbit, and thus we have the apriori estimate

$$
1 / 9 \approx .1111<w<\bar{\sigma} \approx .1231
$$

The only remaining piece of information missing is the initial condition for the shockwave. At first one might think that this initial condition can be chosen arbitrarily, but as we have shown in the last section, the condition that the energy density be positive in front of the shock-wave, or that it be larger than the pressure in front of the shock, puts a constraint on the maximum shock position at a given time. That is, assuming that $w$ lies on the critical orbit implies that the hypotheses of Theorem 5 hold, and thus condition (6.22) is equivalent to $\bar{Q}>0$, and the condition (6.23) is sufficient to guarantee that $\bar{Q}>\bar{P}$, at any given value of $R$. Moreover, if (6.22) or (6.23) hold at a given value $R=R_{*}$, Theorem 5 tells us that they continue to hold for all $R>R_{*}$.

Under the above assumptions, we now obtain estimates for the shock position. To start, rewrite (5.11) as

$$
\begin{aligned}
\frac{d r^{2}}{d R} & =\frac{2(1-w) R}{\alpha\left(3+\frac{\beta}{\alpha} R\right)\left(3+\frac{\beta}{\alpha} R+w\right)} \\
& =\frac{2(1-w) \frac{\alpha}{\beta^{2}} R}{\left(R+\frac{\alpha}{\beta}(3+w)\right)\left(R+3 \frac{\alpha}{\beta}\right)} .
\end{aligned}
$$

Using (7.1) in (7.2) gives the estimate

$$
\frac{\left(1-w_{+}\right) \frac{2 \alpha}{\beta^{2}} R}{\left(R+\left(3+w_{+}\right) \frac{\alpha}{\beta}\right)\left(R+3 \frac{\alpha}{\beta}\right)} \leq \frac{d r^{2}}{d R} \leq \frac{\left(1-w_{-}\right) \frac{2 \alpha}{\beta^{2}} R}{\left(R+\left(3+w_{-}\right) \frac{\alpha}{\beta}\right)\left(R+3 \frac{\alpha}{\beta}\right)}
$$


where $w_{-}=1 / 9 \approx .1111<w_{+}=\bar{\sigma} \approx .1231$. That is,

$$
\frac{\alpha}{\beta^{2}} \frac{2(1-\bar{\sigma}) R}{\left(R+(3+\bar{\sigma}) \frac{\alpha}{\beta}\right)\left(R+3 \frac{\alpha}{\beta}\right)} \leq \frac{d r^{2}}{d R} \leq \frac{\alpha}{\beta^{2}} \frac{(16 / 9) R}{\left(R+\left(\frac{28 \alpha}{9 \beta}\right)\left(R+3 \frac{\alpha}{\beta}\right)\right.}
$$

Now by direct calculation, the solution to the ODE

$$
\frac{d r^{2}}{d R}=C \frac{R}{(R+A)(R+B)},
$$

for positive constants $A, B$, and $C$, is given by

$$
r^{2}=r_{*}^{2}+\ln \left[\left(\frac{R+A}{R_{*}+A}\right)^{\frac{A C}{A-B}}\left(\frac{R+B}{R_{*}+B}\right)^{\frac{-B C}{A-B}}\right],
$$

where inequalities can be substituted for equalities in (7.5), (7.6). Applying this to (7.3) gives the inequalities

$$
\begin{aligned}
& r^{2}-r_{*}^{2} \geq \ln \left[\left(\frac{R+A_{+}}{R_{*}+A_{+}}\right)^{a_{+}}\left(\frac{R+B_{+}}{R_{*}+B_{+}}\right)^{b_{+}}\right], \\
& r^{2}-r_{*}^{2} \leq \ln \left[\left(\frac{R+A_{-}}{R_{*}+A_{-}}\right)^{a_{-}}\left(\frac{R+B_{-}}{R_{*}+B_{-}}\right)^{b_{-}}\right],
\end{aligned}
$$

where

$$
\begin{aligned}
& A=(3+w) \frac{\alpha}{\beta}, \\
& B=3 \frac{\alpha}{\beta}, \\
& a=\frac{2(3+w)(1-w)}{w} \frac{\alpha}{\beta^{2}}, \\
& b=-\frac{6(1-w)}{w} \frac{\alpha}{\beta^{2}},
\end{aligned}
$$

and $A_{-}, A_{+}$, are obtained by substituting $w_{-}, w_{+}$for $w$, respectively, in the above expressions, etc.

We now evaluate $\alpha$ and $\beta$ in terms of the present value of the Hubble constant $H_{0}$ and the observed microwave background radiation temperature $T_{0}$. Here we let subscript zero denote value at present time in the FRW metric, and WLOG we assume that $R_{0}=1$. Recall that the FRW equation (2.5) for $k=0$ can be written as

$$
H^{2} \equiv\left(\frac{\dot{R}}{R}\right)^{2}=Q R^{2}
$$

so that the "Hubble constant" $H_{0}$ is

$$
H_{0}=\sqrt{Q_{0}},
$$


where $Q_{0}$ denotes the present value of the (scaled) energy density in the universe at present time. By (4.13),

$$
Q_{0}=3 \alpha+\beta,
$$

where $3 \alpha$ is the energy density of radiation at present time, and $\beta$ is the energy density of matter at present time. Let $T \equiv T(R)$ denote the temperature of radiation. Then (4.21) is

$$
T=\left(\frac{3 \alpha}{\hat{a}}\right)^{1 / 4} \frac{1}{R}
$$

where (4.20) gives

$$
\hat{a}=\frac{8 \pi \mathcal{G} a}{3 c^{4}} .
$$

Setting $R_{0}=1$ and solving (4.21) for $\alpha$ gives

$$
\alpha=\frac{\hat{a}}{3} T_{0}^{4},
$$

and using this in (4.13) gives

$$
\beta=Q_{0}-3 \alpha=H_{0}^{2}-\hat{a} T_{0}^{4} .
$$

We evaluate the above constants using the values, ([15]),

$$
\begin{aligned}
\frac{\mathcal{G}}{c^{2}} & =7.425 \times 10^{-29} \mathrm{~cm} \mathrm{~g}^{-1}, \\
c & =2.997925 \times 10^{10} \mathrm{~cm} \mathrm{~s}^{-1}, \\
\text { lty } & =9.4605 \times 10^{17} \mathrm{~cm}, \\
\mathrm{mpc} & =10^{6} \mathrm{pc}=3.2615 \times 10^{6} \mathrm{lty}, \\
a & =7.5641 \times 10^{-15} \mathrm{erg} \mathrm{cm}^{-3} \mathrm{~K}^{-4}, \\
H_{0} & =100 h_{0} \mathrm{~km} \mathrm{~s}^{-1} \mathrm{mpc}^{-1}, \\
T_{0} & =2.736^{\circ} \mathrm{K} .
\end{aligned}
$$

Here, $\mathcal{G}$ is Newton's gravitational constant, $c$ the speed of light, lty is lightyear, mpc is megaparcec, ${ }^{\circ} \mathrm{K}$ is degrees Kelvin, $a$ is the Stefan-Boltzmann constant, $T_{0}$ is the observed microwave background radiation temperature [9], and $H_{0}$ is Hubble's constant, where $h_{0}$ is generally accepted to be between .5 and unity. (We take $h_{0} \approx .55$ as a recently quoted value.) Using these values we calculate

$$
\begin{gathered}
\hat{a}=4.6852 \times 10^{-27} \mathrm{lty}^{-2} K^{-4}, \\
H_{0}=1.023 h_{0} \times 10^{-10} \mathrm{lty}^{-1} .
\end{gathered}
$$

Using the above values we obtain from (7.11) and (7.12) that

$$
\frac{\alpha}{\beta}=\frac{1 / 3}{\left(\frac{H_{0}^{2}}{\hat{a} T_{0}^{4}}\right)+1} \approx \frac{\hat{a}}{3 H_{0}^{2}} T_{0}^{4}=\frac{1.492 T_{0}^{4}}{h_{0}^{2}} \times 10^{-7},
$$


and

$$
\begin{aligned}
\frac{\alpha}{\beta^{2}} & =\frac{\hat{a} T_{0}^{4}}{3\left[\hat{a} T_{0}^{4}\left(\frac{H_{0}^{2}}{\hat{a} T_{0}^{4}}\right)-1\right]} \approx\left(\frac{\hat{a}}{3 H_{0}^{2}}\right) \frac{T_{0}^{4}}{H_{0}^{2}} \\
& =\left(1.492 \times 10^{-7}\right) \frac{T_{0}^{4}}{h_{0}^{2} H_{0}^{2}}=\frac{8.34 \times 10^{-6}}{h_{0}^{2} H_{0}^{2}},
\end{aligned}
$$

where we used $\frac{\hat{a} T_{0}^{4}}{H_{0}^{2}}<<1$ at the approximate equality. Using these values we can evaluate:

$$
\begin{aligned}
A_{+} & =(3+.1231) \frac{\alpha}{\beta}=\left(4.66 \times 10^{-7}\right) \frac{T_{0}^{4}}{h_{0}^{2}} \\
B_{+} & =3 \frac{\alpha}{\beta}=\left(4.48 \times 10^{-7}\right) \frac{T_{0}^{4}}{h_{0}^{2}}, \\
a_{+} & =\frac{2(3+.1231)(1-.1231)}{.1231} \frac{\alpha}{\beta^{2}}=\left(6.639 \times 10^{-6}\right) \frac{T_{0}^{4}}{h_{0}^{2} H_{0}^{2}}, \\
b_{+} & =-\frac{6(1-.1231)}{.1231} \frac{\alpha}{\beta^{2}}=\left(6.377 \times 10^{-6}\right) \frac{T_{0}^{4}}{h_{0}^{2} H_{0}^{2}}, \\
A_{-} & =(3+1 / 9) \frac{\alpha}{\beta}=\left(4.64 \times 10^{-7}\right) \frac{T_{0}^{4}}{h_{0}^{2}}, \\
B_{-} & =3 \frac{\alpha}{\beta}=\left(4.48 \times 10^{-7}\right) \frac{T_{0}^{4}}{h_{0}^{2}}, \\
a_{-} & =\frac{2(3+1 / 9)(1-1 / 9)}{1 / 9} \frac{\alpha}{\beta^{2}}=\left(7.427 \times 10^{-6}\right) \frac{T_{0}^{4}}{h_{0}^{2} H_{0}^{2}}, \\
b_{-} & =-\frac{6(1-1 / 9)}{1 / 9} \frac{\alpha}{\beta^{2}}=\left(7.162 \times 10^{-6}\right) \frac{T_{0}^{4}}{h_{0}^{2} H_{0}^{2}} .
\end{aligned}
$$

Now, assuming that the uncoupling of matter and radiation occurred at a temperature less than 4000 degrees Kelvin, [15], it follows from (4.21) that

$$
R_{*} \geq 2.2 / 4000=6.75 \times 10^{-4},
$$

and so it follows that we can essentially neglect the $A$ 's and $B$ 's in estimates (7.7) and (7.8), given their small values in (7.25) and (7.26), and assuming this, estimates (7.7) and (7.8) reduce to,

$$
\left(a_{+}+b_{+}\right) \ln \left(1 / R_{*}\right) \leq r^{2}-r_{*}^{2} \leq\left(a_{-}+b_{-}\right) \ln \left(1 / R_{*}\right) .
$$

Using (7.25) and (7.26) to estimate (7.28) gives the following estimate for the distance the shock-wave must have traveled between $r=R_{*}$ and $R=1$ as predicted by our model:

$$
\frac{\left(2.62 \times 10^{-7}\right) T_{0}^{4}}{h_{0}^{2} H_{0}^{2}} \ln \left(\frac{1}{R_{*}}\right) \leq r^{2}-r_{*}^{2} \leq \frac{\left(2.65 \times 10^{-7}\right) T_{0}^{4}}{h_{0}^{2} H_{0}^{2}} \ln \left(\frac{1}{R_{*}}\right) .
$$


Here the distance $r$ is given in terms of the Hubble length

$$
H_{0}^{-1} \approx \frac{.98}{h_{0}} \times 10^{10}
$$

In particular, (7.29) shows that, in this shock-wave model, the quantity $r^{2}-r_{*}^{2}$ is essentially independent of the starting position $r_{*}$.

As an example, if we take $h_{0}=.55, T_{0}=2.736^{\circ} \mathrm{K}$ and $R_{*}=2.7 / 4000$ in (7.29), we obtain the estimate

$$
r^{2}-r_{*}^{2} \approx\left(\frac{.019}{H_{0}}\right)^{2}
$$

In the standard interpretation of the FRW metric in Cosmology, the galaxies are in freefall, and traverse geodesics $r=$ const. Thus we can interpret $r^{2}-r_{*}^{2}$ in (7.29) as the (squared) distance that the shock-wave travels over and above the motion due to freefall, a result of the fact that mass and momentum are driven across the shock-wave as it evolves outward. We conclude that the distance the shock-wave has traveled, (over and above freefall), between $R=R_{*}=2.7 / 4000$ and $R=1$, as predicted by this model, is approximately .019 of the Hubble length. (Recall that $\bar{r}=R(t) r$ measures distance in lightyears for the three dimensional space at fixed time $t$ in the $k=0$ FRW metric.)

We now discuss the initial condition $r=r_{*}$ at $R=R_{*}$. We saw in (6.23) that the condition $\bar{Q}>\bar{P}$ put constraints on the maximal shock position at each value of $R$. Using the value $\bar{\sigma}=.1231$ in (6.23) gives the inequality

$$
r_{*}^{2}<\frac{.759 R^{2}}{(3.11) \alpha+\beta R}=\frac{.759}{1+\left[\frac{\left(4.64 \times 10^{-7}\right) T_{0}^{4}}{h_{0}^{2} R_{*}^{2}}\right]} \frac{R_{*}}{H_{0}^{2}}
$$

Estimate (7.32) is the bound on the initial shock position, imposed by $\bar{Q}>\bar{P}$, in terms of the Hubble length. Putting (7.32) together with (7.29), we conclude that the maximal distance $r_{\max }$ from the shock-wave to the center of the explosion $r=0$ at present time $R=1$, given as a function of starting time $R_{*}, 2.7 / 4000 \leq R_{*} \leq 1$, (assuming the shock-wave started at position $r=r_{*}$ at $R_{*} \geq 2.7 / 4000$, and such that $r_{*}$ is restricted by (7.32) so that $\bar{Q}>\bar{P}$ for all $R>R_{*}$ ), is predicted by this model to be

$$
r_{\max } \approx H_{0}^{-1} \sqrt{\frac{.76}{1+\left[\frac{\left(4.6 \times 10^{-7}\right) T_{0}^{4}}{h_{0}^{2} R_{*}^{2}}\right]} R_{*}+\left(2.6 \times 10^{-7}\right) \frac{T_{0}^{4}}{h_{0}^{2}} \ln \left(\frac{1}{R_{*}}\right)} .
$$

For example, taking the value $h_{0}=.55$ and $T_{0}=2.736^{\circ} \mathrm{K}$ gives the formula

$$
r_{\max } \approx H_{0}^{-1} \sqrt{\frac{.76}{1+\left[\frac{\left(8.5 \times 10^{-5}\right)}{R_{*}^{2}}\right]} R_{*}+\left(4.9 \times 10^{-5}\right) \ln \left(\frac{1}{R_{*}}\right)}
$$

This function is plotted in Fig. 2. Using (7.33) in (7.29) we obtain the following upper and lower bounds for the shock position $r$ at present time $R=1$ assuming that it starts 


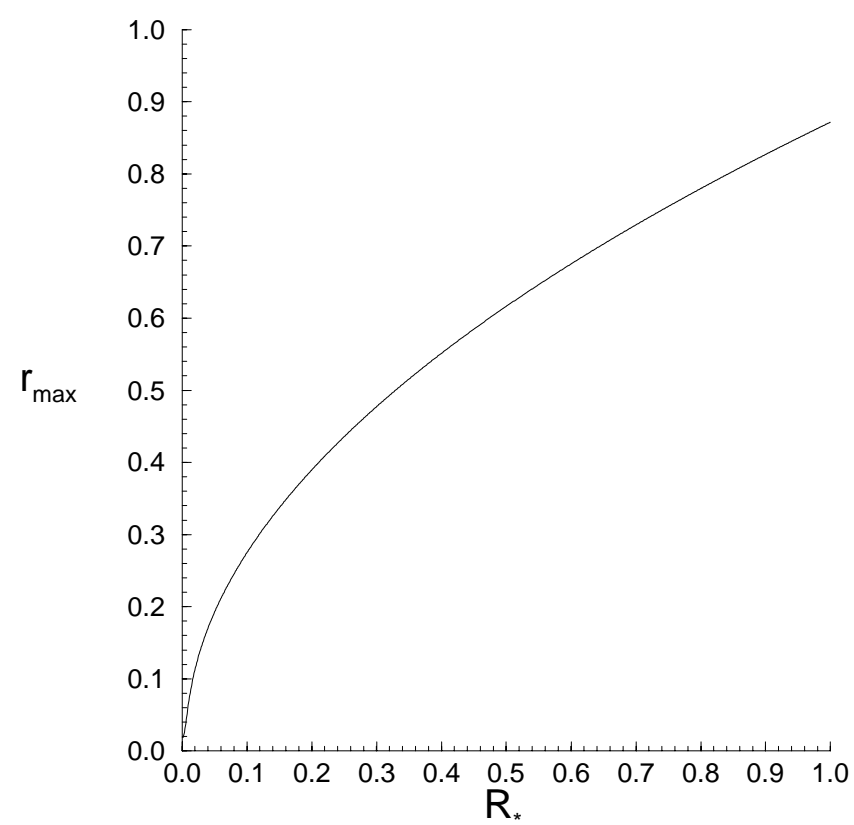

Fig. 2. $r_{\max }$ is in units of $H_{0}^{-1}, H_{0}=100 h_{0} \frac{\mathrm{km}}{\mathrm{smpc}}, h_{0}=.55$

at $R=R_{*}$, and such that $\bar{Q}>\bar{P}$ holds for all $R \geq R_{*}$ :

$$
\begin{aligned}
& r \geq H_{0}^{-1}\left\{\left(5.1 \times 10^{-4}\right) \frac{T_{0}^{2}}{h_{0}} \sqrt{\left.\ln \left(\frac{1}{R_{*}}\right)\right\}},\right. \\
& r \leq H_{0}^{-1} \sqrt{\frac{.76}{1+\left\{\frac{\left(4.6 \times 10^{-7}\right) T_{0}^{4}}{h_{0}^{2} R_{*}^{2}} R_{*}+\left(2.6 \times 10^{-7}\right) \frac{T_{0}^{4}}{h_{0}^{2}} \ln \left(\frac{1}{R_{*}}\right) .\right.}} .
\end{aligned}
$$

\section{The Case of Pure Radiation, $\beta=0$}

As a point of comparison, in this section we redo the calculation of the shock position under the assumption $\beta=0$ in (4.13); that is, under the assumption that the energy density $Q$ is due entirely to radiation, (see [11]. Thus assume that $\alpha=\hat{a} T_{0}^{4} / 3$ is as given in (7.11), but that $\beta=0$. We estimate the position of the shock-wave in this model at the time $R=1$, where $T=T_{0}$. Now of course, since $\beta$ is determined in (7.12) from $H_{0}$ in the above analysis, the value of $\frac{\dot{R}}{R}$ in the pure radiation model will not coincide with $H_{0}$ at the time when $T=T_{0}$. Nevertheless, for comparison purposes, we shall estimate the radial position of the shock-wave in the pure radiation model at time $R=1$ in terms of the Hubble length $H_{0}^{-1}$ given in (7.30). 
In the case $\beta=0$, the constraint (6.23) that guarantees $\bar{Q}>\bar{P}$ reduces to

$$
\alpha r_{*}^{2}<\left(\frac{(1-\bar{\sigma} / 3)(1-w)}{(1+\bar{\sigma})(3+w)}\right) R_{*}^{2}
$$

and the critical orbit becomes $w \equiv \bar{\sigma}$. Using $w=\bar{\sigma} \approx .1231$ in (8.1) gives

$$
r_{*}<\frac{.49}{\sqrt{\alpha}} R_{*} .
$$

(Note that in the alternative case $\alpha=0$, the case of pure matter, the RHS of (6.23) tends to infinity, and thus (6.23) places no constraint on the shock position. This is consistent with the fact that when $\alpha=0$, the pressure is zero, and the shock-wave reduces to a contact discontinuity. For example, $\bar{Q}=0, \bar{P}=0$, solves the shock equations (3.40), (3.41) and it is not difficult to show that the solution of the shock equations in this case reduces to the $k=0$ version of the Oppenheimer-Snyder model, first presented in [11]. In these Oppenheimer-Snyder models, there are no constraints on the shock position corresponding to (6.23).)

Setting $\beta=0$ and $w=\bar{\sigma}$ in (5.11) gives

$$
\frac{d r^{2}}{d R}=\frac{2(1-\bar{\sigma})}{3(3+\bar{\sigma})} \frac{R}{\alpha}
$$

as the differential equation for the shock position. Integrating gives

$$
r^{2}=\frac{(1-\bar{\sigma})}{3(3+\bar{\sigma})} \frac{R^{2}}{\alpha}+r_{*}^{2} .
$$

Using (8.2) for the maximum value of $r_{*}$ yields the following bounds on the shock position $r$ at the time $R=1$ when $T=T_{0}$ that are analogous to (7.35) and (7.36) and apply when $\beta=0$ :

$$
\frac{1}{\sqrt{\alpha}} \sqrt{\frac{(1-\bar{\sigma})}{3(3+\bar{\sigma})}} \leq r \leq \frac{1}{\sqrt{\alpha}} \sqrt{\frac{(1-\bar{\sigma})}{3(3+\bar{\sigma})}+.24 R_{*}} .
$$

From (7.11) it follows that

$$
\frac{1}{\sqrt{\alpha}}=118 h_{0} H_{0}^{-1}
$$

for the value of $H_{0}$ given in (7.18), (note as above that $H_{0} \neq \sqrt{Q_{0}}$ in this case). Using this value together with the value $\bar{\sigma}=.1231$ in (8.5) yields

$$
\frac{36 h_{0}}{H_{0}} \leq r \leq \frac{36 h_{0} \sqrt{1+2.5 R_{*}}}{H_{0}} .
$$

Note that the shock position at $R=1$ that applies to the exact solution given in [11], which was discussed in detail at the end of Sect. 3, is the case $R_{*}=0$ in (8.7). 


\section{References}

1. Blau, S.K. and Guth, A.H.: Inflationary cosmology. In: Three Hundred Years of Gravitation, ed. by S.W. Hawking and W. Israel. Cambridge: Cambridge University Press, 1987, pp. 524-603

2. Hawking, S.W. and Ellis, G.F.R.: The Large Scale Structure of Spacetime. Cambridge: Cambridge University Press, 1973

3. Hawking, S.W. and Penrose, R.: The singularities of gravitational collapse and cosmology. Proc. Roy. Soc. Lond. A 314, 529-548 (1970)

4. Israel, W.: Singular hypersurfaces and thin shells in general relativity. Il. Nuovo Cimento 54B, 1-14 (1966)

5. Lax, P.D.: Shock-waves and entropy. In: Contributions to Nonlinear Functional Analysis, ed. by E. Zarantonello. London-New York: Academic Press, 1971, pp. 603-634

6. Longair, M.S.: Our Evolving Universe. Cambridge: Cambridge University Press, 1996

7. Misner, C., Thorne, K. and Wheeler, J.: Gravitation. San Francisco, CA: Freeman, 1973

8. Oppenheimer, J.R. and Snyder, J.R.: On continued gravitational contraction. Phys. Rev. 56, 455-459 (1939)

9. Peebles, P.J.E.: Principles of Physical Cosmology. Princeton, W: Princeton University Press, 1993

10. Smoller, J. and Temple, B.: Shock-wave solutions of the Einstein equations: The Oppenheimer-Snyder model of gravitational collapse extended to the case of non-zero pressure. Arch. Rat. Mech. Anal. 128, 249-297 (1999)

11. Smoller, J. and Temple, B.: Astrophysical shock-wave solutions of the Einstein equations. Phys. Rev. D 51, No. 6 (1995)

12. Smoller, J. and Temple, B.: General relativistic shock-waves that extend the Oppenheimer-Snyder model. Arch. Rat. Mech. Anal. 138, 239-277 (1997)

13. Smoller, J.: Shock-Waves and Reaction-Diffusion Equations, 2nd ed. Berlin-Heidelberg-New York: Springer-Verlag, 1994

14. Wald, R.M.: General Relativity. Chicago, IL: University of Chicago Press, 1984

15. Weinberg, S.: Gravitation and Cosmology: Principles and Applications of the General Theory of Relativity. New York: Wiley, 1972

Communicated by H. Nicolai 